INSTITUT NATIONAL DE RECHERCHE EN INFORMATIQUE ET EN AUTOMATIQUE

\title{
On the geometry and algebra of the point and line correspondences between $N$ images
}

Olivier Faugeras and Bernard Mourrain

\section{$N^{\circ} 2665$}

October 1995

PROGRAMME 4

\section{apport}

derecherche 



\title{
On the geometry and algebra of the point and line correspondences between $N$ images
}

\author{
Olivier Faugeras and Bernard Mourrain \\ Programme 4 - Robotique, image et vision \\ Projet ROBOTVIS, SAFIR
}

Rapport de recherche $\mathrm{n}^{\circ} 2665$ - October $1995-49$ pages

\begin{abstract}
We explore the geometric and algebraic relations that exist between correspondences of points and lines in an arbitrary number of images. We propose to use the formalism of the GrassmannCayley algebra as the simplest way to make both geometric and algebraic statements in a very synthetic and effective way (i.e. allowing actual computation if needed). We have a fairly complete picture of the situation in the case of points: there are only three types of algebraic relations which are satisfied by the coordinates of the images of a 3-D point: bilinear relations arising when we consider pairs of images among the $N$ and which are the well-known epipolar constraints, trilinear relations arising when we consider triples of images among the $N$, and quadrilinear relations arising when we consider four-tuples of images among the $N$.

Moreover, we show that for a given triple of images, once the epipolar constraints are known, there is only one algebraically independent trilinear relation which can be used to predict the image coordinates of a point in a third image, given the coordinates of the images in the other two images, even in cases where the prediction by the epipolar constraints fails (points in the trifocal plane, or optical centers aligned). We also show that the trilinear relations imply the bilinear ones, i.e. the epipolar constraints. Finally, we show that the quadrilinear relations are algebraically dependent of the trilinear and bilinear ones, i.e. do not bring in any new information.

In the case of lines, we show how the traditional perspective projection equation can be suitably generalized and that in the case of three images there exist two independent trilinear relations between the coordinates of the images of a 3-D line.
\end{abstract}

Key-words: Invariants, Geometry, Geometry of $N$ cameras, Grassmann-Cayley algebra, Plücker relations, multiple cameras stereo.

(Résumé : tsvp)

Original version completed in June 1995, updated October 1995

Unité de recherche INRIA Sophia-Antipolis

2004 route des Lucioles, BP 93, 06902 SOPHIA-ANTIPOLIS Cedex (France)

Téléphone : (33) 93657777 - Télécopie : (33) 93657765 


\section{Sur la géométrie des correspondences entre points et droites dans $N$ images}

Résumé : Nous étudions les relations algébriques et géométriques entre les correspondences de points et de droites dans un nombre arbitraire d'images. Nous proposons pour ce faire d'utiliser le formalisme de l'algèbre de Grassmann-Cayley qui nous apparaît comme l'un des moyens les plus simples pour exprimer de manière effective (c'est-à-dire permettant les calculs) des propriétés géométriques et algébriques.

La situation dans le cas des points est assez bien comprise. Il n'y a que trois types de relations algébriques sur les coordonnées images d'un point $3-\mathrm{D}$ : des relations bilinéaires apparaîssent lorsqu'on considère des couples d'images et induisent les contraintes épipolaires, bien connues; des relations trilinéaires apparaîssent lorsque l'on considère des triplets d'images, et des relations quadrilinéaires apparaîssent lorsque l'on considère des quadruplets d'images.

De plus nous montrons que pour un triplet d'images donné, lorsque les contraintes épipolaires sont connues, il n'y a qu'une relation trilinéaire algébriquement indépendante et qui peut être utilisée pour prédire les coordonnées d'un point dans la troisième image à partir des coordonnées des deux autres, ceci même dans les cas où la géométrie épipolaire ne permet pas de le faire (points dans le plan trifocal, centres optiques alignés). Nous monstrons aussi que les relations trilinéaires impliquent les bilinéaires, c'est-à-dire les contraintes épipolaires. Enfin nous montrons que les relations quadrilinéaires sont algébriquement dépendantes des relations bilinéaires et trilinéaires, et donc n'apportent pas a priori d'information supplémentaire.

Dans le cas des droites nous montrons que l'équation classique de projection perspective peut être généralisée convenablement et que dans le cas de trois images il existe deux relations trilinéaires indépendantes entre les coordonnées des images d'une droite 3-D.

Mots-clé : Invariants, Géometrie, Géometrie de $N$ caméras, algèbre de GrassmannCayley, relations de Plücker, stéréoscopie. 


\section{Introduction}

Understanding the geometry of the correspondences between image primitives that arise from the perspective projection of three-dimensional objects is fundamental for such applications as three-dimensional reconstruction from multiple views, for example stereo and motion, object recognition, image synthesis, image coding. Recent theoretical efforts directed toward the development of such an understanding have demonstrated the importance of projective geometry as the language allowing the simplest description of the underlying phenomena.

In the case of two cameras, the theory is almost complete, the main fact being that correspondences between points in two images are completely described by the epipolar geometry which can itself be summarized algebraically in a $3 \times 3$ matrix of rank 2 , the fundamental matrix [17, 18, 19]. Moreover, it has been shown by several authors $[9,13,26]$ that once the fundamental matrix is known, and correspondences between points established, the 3-D scene imaged by the two cameras can be reconstructed up to a projective transformation. The case of three images or more has not been studied as extensively as the case of two. Faugeras and Robert [8] have shown that the knowledge of the three fundamental matrixes of the three pairs of images could be used to predict correspondences in a third image from correspondences in the other two by a simple use of the epipolar geometry. We show in the main body of this article that this method may fail in some cases (see also [31]).

A closely related problem is that of determining the number of degrees of freedom of the set of the three fundamental matrixes of three views: can they be arbitrary or do they have to satisfy some constraints? In $[16,8]$ the answer is shown to be that they cannot be chosen arbitrarily and that they must satisfy three algebraic constraints, reducing the number of free parameters from 21 to 18. An alternative to this approach based on the epipolar geometry has been proposed by Hartley, Shashua and Werman [25, 12, 27] who have introduced third-degree algebraic constraints on the coordinates of the three images of a generic 3-D point or line which can also be used to predict coordinates in a third image from the coordinates in the other two. These algebraic constraints are called Shashua's trilinearities or Hartley's trifocal tensor. Hartley has shown that they were identical. There are a number of questions related to 
this tensor which have been left partly unanswered by the previous two authors and which we address in this paper. These are the following: is this tensor unique or are there other tensors? Does it have to satisfy algebraic constraints like the fundamental matrixes? Does it provide a solution to the transfer problem when the epipolar method fails?

We answer these questions and others by proposing a systematic way of deriving the necessary and sufficient algebraic relations which have to be satisfied by the coordinates of image points in an arbitrary number of images in order for them to be the images of a single 3-D point. We show that these relationships are of three types only:

Bilinear: This is the well-known epipolar condition provided by the fundamental matrix.

Trilinear: These are the trilinear relations of which there are a priori 12 for three views among which two only are algebraically independent once we take into account the epipolar constraints.

Quadrilinear: These are the quadrilinear relations which appear when the number of views reaches four.

We describe the algebraic relations between these three classes of relations as well as the algebraic relations which must be satisfied by the coefficients of the trilinear ones.

We also investigate the case of line correspondences. The analysis is made a bit more complicated than in the case of points because the perspective projection equation has been developed for points. We show that this equation can be very simply extended to lines by representing them with their Plücker coordinates. The perspective projection matrix for lines is shown to be a $3 \times 6$ matrix whose rows are the Plücker coordinates of the lines of intersection of the three planes defined by the row vectors of the perspective projection matrix for points.

This representation allows us to extend in a fairly simple way the analysis which has been done for points. The salient result is the existence of two independent trilinear relations between the coordinates of the images of a 3-D line in three views. These two trilinear relations are the formal analog of the epipolar constraint that applies to the images of a 3-D point in two views. 
The thrust of the paper is to show that all these relations can be derived systematically and very simply from the pinhole camera model using the tools of the Grassmann-Cayley algebra. This, we believe, establishes the adequate theoretical framework for analysing the geometry of the correspondences between $N$ views. We start demonstrating this claim by answering some of the questions related to their uniqueness and the number of their degrees of freedom.

\section{Notations and definitions}

We use projective geometry in all this article. An elementary introduction can be found in [24, 7]. Capital letters indicate 3-D entities, lower case letters 2-D or image entities. Bold letters indicate vectors or matrixes. For example, $M$ represents a $3-\mathrm{D}$ projective point and $\mathbf{M}$ one of its $4 \times 1$ coordinate vectors, $m_{i}$ is the image of $M$ in the $i$-th camera, $\mathbf{m}_{i}$ one of its $3 \times 1$ coordinate vectors of coordinates $x_{i}, y_{i}, z_{i}$. The $i$-th perspective projection matrix is noted $\mathbf{P}_{i}$, a $3 \times 4$ matrix whose 3 row vectors will be denoted by the digits $3 *(i-1)+j, i=$ $1, \cdots, N, j=1,2,3$. The perspective projection equation of the first camera can thus be written as:

$$
\mathbf{m}_{1}=\mathbf{P}_{1} \mathbf{M}=\left[\begin{array}{l}
\mathbf{1} \\
\mathbf{2} \\
\mathbf{3}
\end{array}\right] \mathbf{M}
$$

or

$$
x_{1}: y_{1}: z_{1}=\mathbf{1} \cdot \mathbf{M}: \mathbf{2} \cdot \mathbf{M}: \mathbf{3} \cdot \mathbf{M}
$$

where for example $\mathbf{1} \cdot \mathbf{M}$ represents the usual inner product of the row vector $\mathbf{1}$ with the column vector $\mathbf{M}$. This equation is equivalent to the three (linearly dependent) equations

$$
\begin{aligned}
& x_{1} \mathbf{2} \cdot \mathbf{M}-y_{1} \mathbf{1} \cdot \mathbf{M}=0 \\
& y_{1} \mathbf{3} \cdot \mathbf{M}-z_{1} \mathbf{2} \cdot \mathbf{M}=0 \\
& z_{1} \mathbf{1} \cdot \mathbf{M}-x_{1} \mathbf{3} \cdot \mathbf{M}=0
\end{aligned}
$$

$\operatorname{RR} \mathrm{n}^{\circ} 2665$ 
Finally, given four row or column vectors $\mathbf{U}_{i}, i=1, \cdots, 4$ of size four, the notation $\left[\mathbf{U}_{1}, \mathbf{U}_{2}, \mathbf{U}_{3}, \mathbf{U}_{4}\right]$ will denote the $4 \times 4$ determinant of these four vectors, their bracket.

Let us denote by $\mathbb{E}_{n}$ the vector space of dimension $n$ on the real numbers. We will be using both the cross-product of two vectors $\mathbf{x}$ and $\mathbf{y}$ of $\mathbb{E}_{3} \equiv \mathbb{R}^{3}$, noted $\mathbf{x} \times \mathbf{y}$, a vector of $\mathbb{E}_{3}$ and the exterior product of two vectors of $\mathbb{E}_{n}$, noted $\mathbf{x} \wedge \mathbf{y}$, a vector of $\stackrel{2}{\wedge} \mathbb{E}_{n}$ which is a vector space of dimension $\left(\begin{array}{c}n \\ 2\end{array}\right)$, i.e. also 3 for $n=3$. The projective space associated to $\mathbb{E}_{n+1}$ will be denoted by $\mathbb{P}^{n}$. We assume that the reader is familiar with the basic results of the exterior calculus and the Grassmann-Cayley algebra. A good introduction, targeted at computer vision researchers, can be found in [3], more details can be found in the quite accessible book by Sturmfels [28], and in the more advanced article by Barnabei et al. [2]. A very brief summary of the material required to read this article is given in Appendix B.

\section{Bilinear constraints}

Let us start with the case of two cameras. Even though this case is now well understood, it is worthwhile going through its analysis because it turns out that the way we approach it is rather different from the usual way and can be extended in a straightforward fashion to the trilinear and quadrilinear cases. Let us choose the first two equations among the three equations (2) for each of the two cameras. These four equations can be written in matrix form as:

$$
\left[\begin{array}{l}
x_{1} \mathbf{2}-y_{1} \mathbf{1} \\
y_{1} \mathbf{3}-z_{1} \mathbf{2} \\
x_{2} \mathbf{5}-y_{2} \mathbf{4} \\
y_{2} \mathbf{6}-z_{2} \mathbf{5}
\end{array}\right] \mathbf{M}=\mathbf{0}
$$

The $4 \times 4$ matrix which appears in this equation is a linear function of the two points $m_{1}$ and $m_{2}$. The question is, what is the necessary and sufficient condition that the two points $m_{1}$ and $m_{2}$ must satisfy so that they can be considered as the images of the same point $M$. The answer is well known and is that they must satisfy the epipolar constraint $[1,15,7,11]$. 
Looking at equations (3) we see that the condition we seek is that the $4 \times 4$ matrix must be of rank less than or equal to 3 which is equivalent to writing that its determinant must be equal to 0. Expanding this determinant, we find

$$
y_{1} y_{2}\left(\mathbf{m}_{2}^{T} \mathbf{F}_{1,2} \mathbf{m}_{1}\right)
$$

where $\mathbf{F}_{1,2}$ is a $3 \times 3$ matrix which we recognize as the fundamental matrix of the first two images $[17,10,30]$.

Its elements are $4 \times 4$ determinants built from the row vectors of the perspective projection matrices $\mathbf{P}_{1}$ and $\mathbf{P}_{2}$. In detail

$$
\mathbf{F}_{1,2}=\left[\begin{array}{rrr}
{[2,3,5,6]} & -[1,3,5,6] & {[1,2,5,6]} \\
-[2,3,4,6] & {[1,3,4,6]} & -[1,2,4,6] \\
{[2,3,4,5]} & -[1,3,4,5] & {[1,2,4,5]}
\end{array}\right] .
$$

the factor $y_{1} y_{2}$ comes from the choice of the specific equations among (2) to build the determinant. Another choice of equation will produce another factor but the matrix $\mathbf{F}_{1,2}$ will remain unchanged. Note that this constraint can also be rewritten in a symmetric way, using some standard tools of the GrassmannCayley algebra described in the appendix B:

$$
\left(x_{1} \mathbf{2} \wedge \mathbf{3}+y_{1} \mathbf{3} \wedge \mathbf{1}+z_{1} \mathbf{1} \wedge \mathbf{2}\right) \wedge\left(x_{2} \mathbf{5} \wedge \mathbf{6}+y_{2} \mathbf{6} \wedge 4+z_{2} 4 \wedge 5\right)=0
$$

which also explains why it is independent of the choice of the equations. This constraint can be interpreted geometrically as follows. The vectors $\mathbf{1 , 2}, \mathbf{3}$ represent planes through the optical center of the first camera. So $\mathbf{2} \wedge \mathbf{3}, \mathbf{1} \wedge$ $\mathbf{3}, \mathbf{1} \wedge \mathbf{2}$ are lines through this center and $\left(x_{1} \mathbf{2} \wedge \mathbf{3}+y_{1} \mathbf{3} \wedge \mathbf{1}+z_{1} \mathbf{1} \wedge \mathbf{2}\right)$ is just the optical ray from the center to the point $M$ or $m_{1}$. The equation (5) corresponds to the fact that the rays from the center of the two cameras have a common point $M$ in the space $\mathbb{P}^{3}$.

The fact that the fundamental matrix $\mathbf{F}_{1,2}$ is of rank less than or equal to two can be found in several ways. The first way is to expand its determinant as a function of the brackets $[i, j, k, l]$ and use a straightening algorithm to rewrite it in standard form (see $[28,23]$ ). The result is found to be the null polynomial. The second way is to show that there are scalars $\lambda_{i}, i=1,2,3$ such that $\lambda_{1} \mathbf{f}_{1}+\lambda_{2} \mathbf{f}_{2}+\lambda_{3} \mathbf{f}_{3}=\mathbf{0}$ where the $\mathbf{f}_{i}$ 's are the column vectors of $\mathbf{F}_{1,2}$. This is done explicitly in the next paragraph. 
The epipoles can also be found quite easily. One way is to find values of $x_{1}, y_{1}, z_{1}$ such that the line $\left(x_{1} \mathbf{2} \wedge \mathbf{3}+y_{1} \mathbf{3} \wedge \mathbf{1}+z_{1} \mathbf{1} \wedge \mathbf{2}\right)$ contains the center of the second camera which is the intersection $4 \wedge \mathbf{5} \wedge \mathbf{6}$ of the three planes. Using the standard operators of intersection and sum in the Cayley Algebra (see $[2,22]$ or Appendix B), this yields

$$
\begin{aligned}
& \left(x_{1}[2,4,5,6]-y_{1}[1,4,5,6]\right) \mathbf{3} \\
& \quad-\left(x_{1}[3,4,5,6]-z_{1}[1,4,5,6]\right) \mathbf{2}+\left(y_{1}[3,4,5,6]-z_{1}[2,4,5,6]\right) \mathbf{1}=0
\end{aligned}
$$

As the vectors $\mathbf{1}, \mathbf{2}, \mathbf{3}$ are independent, we obtain a system of 3 (dependent) equations, which determines the epipole $e_{1,2}$.

Another way to compute the coordinates of the epipole $e_{1,2}$ in the first image is to use the cross-product of any two row vectors of $\mathbf{F}_{1,2}$. Computing the cross-product of the first two rows, we find a vector whose coordinates are quadratic polynomials of the brackets. Rewriting these polynomials in standard form, we find that $\mathbf{e}_{1,2}$ is proportional to the vector

$$
[[1,4,5,6],[2,4,5,6],[3,4,5,6]]^{T}
$$

Similarly, the epipole $e_{2,1}$ in the second image is found to be represented by the vector

$$
[[1,2,3,4],[1,2,3,5],[1,2,3,6]]^{T}
$$

These vectors can be also be interpreted as follows: the coordinates of the image $m_{1}$ of a point $M$ in the first camera are given by $[\mathbf{1} \cdot \mathbf{M}, \mathbf{2} \cdot \mathbf{M}, \mathbf{3} \cdot \mathbf{M}]^{T}$. As the epipole $e_{1,2}$ is the image of the center of the second camera defined by $\mathbf{4} \wedge \mathbf{5} \wedge \mathbf{6}$, the coordinates of $e_{1,2}$ are

$$
\begin{gathered}
{[\mathbf{1} \cdot \mathbf{4}} \\
\quad=[\mathbf{5} \wedge \mathbf{6}, \mathbf{2} \cdot \mathbf{4} \wedge \mathbf{5} \wedge \mathbf{6}, \mathbf{3} \cdot \mathbf{4} \wedge \mathbf{5} \wedge \mathbf{6}]^{T} \\
\quad=[1,4,5,6],[2,4,5,6],[3,4,5,6]]^{T} .
\end{gathered}
$$

One can check that $\mathbf{F}_{1,2} \mathbf{e}_{1,2}=0$ :

$>$ evalm(F12 \&* E12);

$$
\left[\begin{array}{r}
{[2,3,5,6][1,4,5,6]-[1,3,5,6][2,4,5,6]+[1,2,5,6][3,4,5,6]} \\
-[2,3,4,6][1,4,5,6]+[1,3,4,6][2,4,5,6]-[1,2,4,6][3,4,5,6] \\
{[2,3,4,5][1,4,5,6]-[1,3,4,5][2,4,5,6]+[1,2,4,5][3,4,5,6]}
\end{array}\right]
$$


$>\operatorname{map}($ stgt, $")$;

$$
\left[\begin{array}{lll}
0 & 0 & 0
\end{array}\right]
$$

These computations have been done in MAPLE. The function stgt that we have implemented (see [23]) gives a normal form to any polynomial in the determinants of 4 points. This normal form is zero if and only if the polynomial is a consequence of the Plücker relations. This evaluation shows that

$$
[1,4,5,6] \mathbf{f}_{1}+[2,4,5,6] \mathbf{f}_{2}+[3,4,5,6] \mathbf{f}_{3}=\mathbf{0}
$$

\section{Trilinear constraints}

Let us now add a third image. Equation (3) can be written as

$$
\left[\begin{array}{l}
x_{1} \mathbf{2}-y_{1} \mathbf{1} \\
y_{1} 3-z_{1} 2 \\
x_{2} 5-y_{2} 4 \\
y_{2} 6-z_{2} 5 \\
x_{3} 8-y_{3} 7 \\
y_{3} \mathbf{9}-z_{3} 8
\end{array}\right] \mathbf{M}=\mathbf{0}
$$

where we have again chosen arbitrarily the first two equations in (2) for each camera. The results to come are independent of this choice.

The $6 \times 4$ matrix which appears in this equation is a linear function of the three points $m_{1}, m_{2}, m_{3}$. The question is, what is the necessary and sufficient condition that the three points $m_{i}$ must satisfy so that they can be considered as the images of the same point $M$. The answer comes again from elementary linear algebra and is that the $6 \times 4$ matrix must be of rank less than or equal to three. This is equivalent to writing that all its $4 \times 4$ subdeterminants are zero. There are two types of such determinants:

1. Those which contain two rows arising from one camera and two rows from another.

2. Those which contain two rows arising from one camera, one row from a second camera and one row arising from the third camera.

$\operatorname{RR} \mathrm{n}^{\circ} 2665$ 
There are three determinants of the first type and, as seen in the previous section, they correspond to the three fundamental matrixes of the three pair of cameras. There are 12 determinants of the second type. Computing for example the one built from the first two rows, the third and the fifth and dividing by $y_{1}$, we find the following expression

$$
\begin{aligned}
x_{1} & x_{2} x_{3}[2,3,5,8]-x_{1} x_{2} y_{3}[2,3,5,7]+x_{1} y_{2} x_{3}[2,4,3,8] \\
- & -x_{1} y_{2} y_{3}[2,4,3,7]-y_{1} x_{2} x_{3}[1,3,5,8]+y_{1} x_{2} y_{3}[1,3,5,7] \\
- & y_{1} x_{3} y_{2}[1,4,3,8]+y_{1} y_{2} y_{3}[1,4,3,7]+z_{1} x_{2} x_{3}[1,2,5,8] \\
& -z_{1} x_{2} y_{3}[1,2,5,7]-z_{1} y_{2} x_{3}[1,2,4,8]+z_{1} y_{2} y_{3}[1,2,4,7]
\end{aligned}
$$

Just as in the case of the bilinear constraints, the factor $y_{1}$ is irrelevant and comes only from the choice of the two equations for the first camera. The other factor is seen to be a polynomial of total degree three in the coordinates of the points $m_{i}$ and linear in the coordinates of each of them. It is a trilinear function analog to those discussed by Shashua and Hartley [25, 12]. It has only 12 nonzero coefficients. If we keep the first two rows fixed, there are four such determinants, hence a total of 12 trilinearities. Let us denote by $T_{i, j, k, l}\left(m_{1}, m_{2}, m_{3}\right)$ such a trilinearity obtained with the rows $i, j, k, l$ of the matrix appearing in the left handside of (6).

A geometric interpretation of this result can be obtained as follows. The line $x_{1} \mathbf{2} \wedge \mathbf{3}+y_{1} \mathbf{3} \wedge \mathbf{1}+z_{1} \mathbf{1} \wedge \mathbf{2}$ is the optical ray of the point $m_{1}$ in the first camera. Similarly $x_{2} 5-y_{2} 4$ (resp. $x_{3} 8-y_{3} 7$ ) is a line in the second (resp. third) retinal plane or a plane through the center of the second (resp. third) camera in $\mathbb{P}^{3}$. More precisely, the first plane (resp. the second) is the plane containing the optical ray of $m_{2}$ (resp. $m_{3}$ ) and the line of intersection of the two planes 4 and 5 (resp. 7 and 8 ). To see this for the first plane, it is sufficient to write $x_{2}=\mathbf{M} \cdot \mathbf{4}$ and $y_{2}=\mathbf{M} \cdot \mathbf{5}$. Note that the line of intersection of those two planes goes through the point $M$. The trilinearity condition is just the condition that this line and these two planes have a common point in $\mathbb{P}^{3}$. Algebraically, this is written as

$T_{1,2,3,5}\left(m_{1}, m_{2}, m_{3}\right)=\left(x_{1} \mathbf{2} \wedge \mathbf{3}+y_{1} \mathbf{3} \wedge \mathbf{1}+z_{1} \mathbf{1} \wedge \mathbf{2}\right) \wedge\left(x_{2} \mathbf{5}-y_{2} \mathbf{4}\right) \wedge\left(x_{3} \mathbf{8}-y_{3} \mathbf{7}\right)$

This is shown in figure 1. Note that the condition $T_{1,2,3,5}=0$ does not imply that the three optical rays intersect at a point, in fact they are in general skew. 
We analyse in detail the geometric interpretation of the trilinearities in section 4.2 .

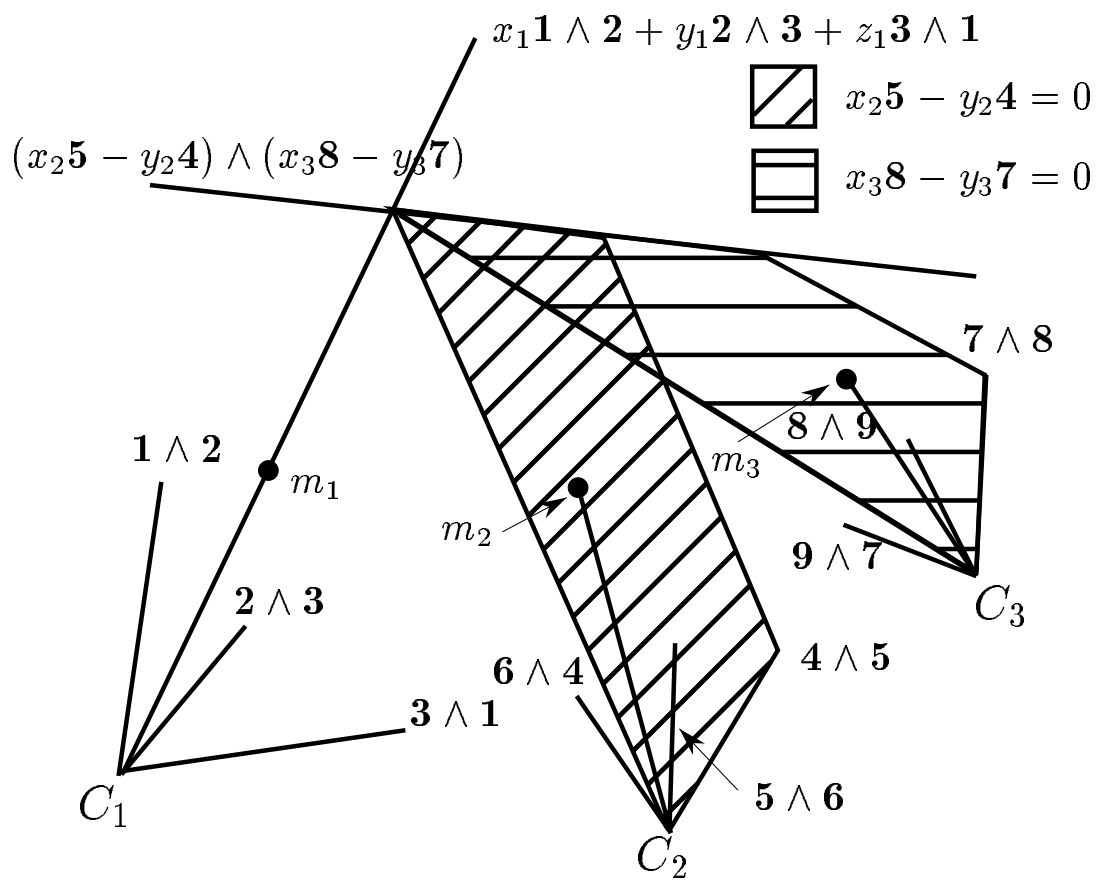

Figure 1: The trilinearity $T_{1,2,3,5}$ expresses the fact that the optical ray $x_{1} \mathbf{2} \wedge$ $\mathbf{3}+y_{1} \mathbf{3} \wedge \mathbf{1}+z_{1} \mathbf{1} \wedge \mathbf{2}$ intersect the line of intersection of the two planes $x_{2} \mathbf{5}-y_{2} \mathbf{4}$ and $x_{3} 8-y_{3} 7$.

All these trilinear relations and the epipolar constraints or bilinear relations are not independent. For example, it is known [8] that the bilinearities satisfy the following three relations:

$$
\mathbf{e}_{i+1, i-1}^{T} \mathbf{F}_{i, i+1} \mathbf{e}_{i, i-1}=0 \text { for } i=1,2,3
$$

where the values of the indexes are computed in the obvious manner.

We now analyse the relations between the trilinearities and the bilinearities. 


\subsection{Algebraic relations between trilinearities and bili- nearities}

It is possible to completely describe the set of algebraic relations between the trilinearities and the fundamental matrices.

It is convenient to classify the trilinearities into three groups, depending upon which camera plays a different role. Using the matrix which appears in equation (6) and the compact notation of equation (7), we put in the first group, those trilinearities built from the first two rows of matrix (6), in the second group those trilinearities built from the third and the fourth rows of (6), and in the third group those trilinearities built from the fifth and the sixth rows of (6). In detail: The relations between these trilinearities and the binary

$$
\begin{aligned}
& \text { Group I } \\
& T_{1,2,3,5}=\left(x_{1} \mathbf{2} \wedge \mathbf{3}+y_{1} \mathbf{3} \wedge \mathbf{1}+z_{1} \mathbf{1} \wedge \mathbf{2}\right) \wedge\left(x_{2} \mathbf{5}-y_{2} \mathbf{4}\right) \wedge\left(x_{3} \mathbf{8}-y_{3} \mathbf{7}\right) \\
& T_{1,2,4,5}=\left(x_{1} \mathbf{2} \wedge \mathbf{3}+y_{1} \mathbf{3} \wedge \mathbf{1}+z_{1} \mathbf{1} \wedge \mathbf{2}\right) \wedge\left(y_{2} \mathbf{6}-z_{2} \mathbf{5}\right) \wedge\left(x_{3} \mathbf{8}-y_{3} \mathbf{7}\right) \\
& T_{1,2,3,6}=\left(x_{1} \mathbf{2} \wedge \mathbf{3}+y_{1} \mathbf{3} \wedge \mathbf{1}+z_{1} \mathbf{1} \wedge \mathbf{2}\right) \wedge\left(x_{2} \mathbf{5}-y_{2} \mathbf{4}\right) \wedge\left(y_{3} \mathbf{9}-z_{3} \mathbf{8}\right) \\
& T_{1,2,4,6}=\left(x_{1} \mathbf{2} \wedge \mathbf{3}+y_{1} \mathbf{3} \wedge \mathbf{1}+z_{1} \mathbf{1} \wedge \mathbf{2}\right) \wedge\left(y_{2} \mathbf{6}-z_{2} \mathbf{5}\right) \wedge\left(y_{3} \mathbf{9}-z_{3} \mathbf{8}\right) \\
& \text { Group II } \\
& T_{1,3,4,5}=\left(x_{1} \mathbf{2}-y_{1} \mathbf{1}\right) \wedge\left(x_{2} \mathbf{4} \wedge \mathbf{5}+y_{2} \mathbf{5} \wedge \mathbf{6}+z_{2} \mathbf{6} \wedge \mathbf{4}\right) \wedge\left(x_{3} \mathbf{8}-y_{3} \mathbf{7}\right) \\
& T_{2,3,4,5}=\left(y_{1} \mathbf{3}-z_{1} \mathbf{2}\right) \wedge\left(x_{2} \mathbf{4} \wedge \mathbf{5}+y_{2} \mathbf{5} \wedge \mathbf{6}+z_{2} \mathbf{6} \wedge \mathbf{4}\right) \wedge\left(x_{3} \mathbf{8}-y_{3} \mathbf{7}\right) \\
& T_{1,3,4,6}=\left(x_{1} \mathbf{2}-y_{1} \mathbf{1}\right) \wedge\left(x_{2} \mathbf{4} \wedge \mathbf{5}+y_{2} \mathbf{5} \wedge \mathbf{6}+z_{2} \mathbf{6} \wedge \mathbf{4}\right) \wedge\left(y_{3} \mathbf{9}-z_{3} \mathbf{8}\right) \\
& T_{2,3,4,6}=\left(y_{1} \mathbf{3}-z_{1} \mathbf{2}\right) \wedge\left(x_{2} \mathbf{4} \wedge \mathbf{5}+y_{2} \mathbf{5} \wedge \mathbf{6}+z_{2} \mathbf{6} \wedge \mathbf{4}\right) \wedge\left(y_{3} \mathbf{9}-z_{3} \mathbf{8}\right) \\
& \text { Group III } \\
& \begin{array}{l}
T_{1,3,5,6}=\left(x_{1} \mathbf{2}-y_{1} \mathbf{1}\right) \wedge\left(x_{2} \mathbf{5}-y_{2} \mathbf{4}\right) \wedge\left(x_{3} \mathbf{7} \wedge \mathbf{8}+y_{3} \mathbf{8} \wedge \mathbf{9}+z_{3} \mathbf{9} \wedge \mathbf{7}\right) \\
T_{1, \mathbf{4}, 5,6}=\left(x_{1} \mathbf{2}-y_{1} \mathbf{1}\right) \wedge\left(y_{2} \mathbf{6}-z_{2} \mathbf{5}\right) \wedge\left(x_{3} \mathbf{7} \wedge \mathbf{8}+y_{3} \mathbf{8} \wedge \mathbf{9}+z_{3} \mathbf{9} \wedge \mathbf{7}\right) \\
T_{2,3,5,6}=\left(y_{1} \mathbf{3}-z_{1} \mathbf{2}\right) \wedge\left(x_{2} \mathbf{5}-y_{2} \mathbf{4}\right) \wedge\left(x_{3} \mathbf{7} \wedge \mathbf{8}+y_{3} \mathbf{8} \wedge \mathbf{9}+z_{3} \mathbf{9} \wedge \mathbf{7}\right) \\
T_{2, \mathbf{4}, 5,6}=\left(y_{1} \mathbf{3}-z_{1} \mathbf{2}\right) \wedge\left(y_{2} \mathbf{6}-z_{2} \mathbf{5}\right) \wedge\left(x_{3} \mathbf{7} \wedge \mathbf{8}+y_{3} \mathbf{8} \wedge \mathbf{9}+z_{3} \mathbf{9} \wedge \mathbf{7}\right)
\end{array}
\end{aligned}
$$

Table 1: Definitions of the trilinearities.

relations are easily found to fall into two categories, the intra-group relations 
of the type of equation (8) and the inter-group relations of the type of equation (9).

All possible relations are summarized in figure 2 where the intra-groups relations are represented by three types of lines and the inter-groups by a fourth type.

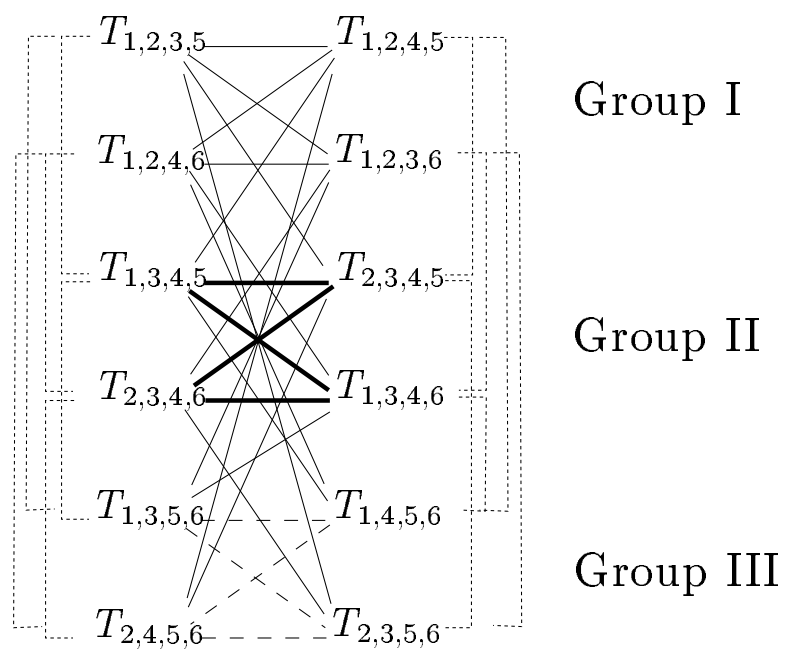

Figure 2: The relations between the 12 trilinearities fall into two categories, intra- and inter-relations represented in the figure by different types of lines.

The simplest way of explaining the method to obtain algebraic relations between trilinearities and bilinearities and in general is to use Cramer's relations (see appendix A) between the row vectors of the matrix appearing in equation (6).

Let us for example derive the relation between $T_{1,2,3,5}, T_{1,2,4,5}$, and $\mathbf{F}_{1,2}$. We consider the row vectors $\mathbf{C}_{1}, \mathbf{C}_{2}, \mathbf{C}_{3}, \mathbf{C}_{4}, \mathbf{C}_{5}$ which are related by the Cramer's relation:

$$
\begin{aligned}
& {\left[\mathbf{C}_{2}, \mathbf{C}_{3}, \mathbf{C}_{4}, \mathbf{C}_{5}\right] \mathbf{C}_{1}-\left[\mathbf{C}_{1}, \mathbf{C}_{3}, \mathbf{C}_{4}, \mathbf{C}_{5}\right] \mathbf{C}_{2}+\left[\mathbf{C}_{1}, \mathbf{C}_{2}, \mathbf{C}_{4}, \mathbf{C}_{5}\right] \mathbf{C}_{3}-\left[\mathbf{C}_{1}, \mathbf{C}_{2}, \mathbf{C}_{3}, \mathbf{C}_{5}\right] \mathbf{C}_{4}+} \\
& {\left[\mathbf{C}_{1}, \mathbf{C}_{2}, \mathbf{C}_{3}, \mathbf{C}_{4}\right] \mathbf{C}_{5}=\mathbf{0}}
\end{aligned}
$$


Multiplying this equation by $\mathbf{C}_{1} \wedge \mathbf{C}_{2} \wedge \mathbf{7}$ we obtain the following relation, typical of the intra-relations within groups:

$T_{1,2,4,5} \operatorname{coeff}\left(T_{1,2,3,5}, y_{3}\right)-T_{1,2,3,5} \operatorname{coeff}\left(T_{1,2,4,5}, y_{3}\right)-x_{3} y_{2}\left(\mathbf{m}_{2}^{T} \mathbf{F}_{1,2} \mathbf{m}_{1}\right)\left(\mathbf{F}_{1,3} \mathbf{m}_{1}\right)_{3}=0$

If we multiply by $\mathbf{C}_{1} \wedge \mathbf{C}_{4} \wedge \mathbf{7}$ we obtain a relation between $T_{1,2,3,5}$ and $T_{1,3,4,5}$, typical of the inter-relations between groups:

$\operatorname{coeff}\left(T_{1,3,4,5}, y_{3}\right) T_{1,2,3,5}-\operatorname{coeff}\left(T_{1,2,3,5}, y_{3}\right) T_{1,3,4,5}-x_{3} \operatorname{coeff}\left(T_{1,3,5,6}, z_{3}\right)\left(\mathbf{m}_{2}^{T} \mathbf{F}_{1,2} \mathbf{m}_{1}\right)=0$

All intra- and inter-relations for the group I are summarized in the table 2. They are obtained by applying Cramer's rule to the points $\left(\mathbf{C}_{1}, \mathbf{C}_{2}, \mathbf{C}_{3}, \mathbf{C}_{4}, \mathbf{C}_{5}\right)$, $\left(\mathbf{C}_{1}, \mathbf{C}_{2}, \mathbf{C}_{3}, \mathbf{C}_{4}, \mathbf{C}_{6}\right),\left(\mathbf{C}_{1}, \mathbf{C}_{2}, \mathbf{C}_{3}, \mathbf{C}_{5}, \mathbf{C}_{6}\right)$, and $\left(\mathbf{C}_{1}, \mathbf{C}_{2}, \mathbf{C}_{4}, \mathbf{C}_{5}, \mathbf{C}_{6}\right)$.

Let us comment on these relations a little, more will be said later about their geometric interpretation. For example, let is consider $T_{1,2,3,5}$ and $T_{1,2,4,5}$. If the pixels $m_{1}$ and $m_{2}$ satisfy the epipolar constraint $\left(\mathbf{m}_{2}^{T} \mathbf{F}_{1,2} \mathbf{m}_{1}=0\right)$, then the first equation in table 2 shows that the two trilinearities are algebraically dependent, i.e. that if one is zero for some triple of points $\left(m_{1}, m_{2}, m_{3}\right)$, then the other one is in general also 0 for the same triple of points. The words "in general" mean, for example, that if $T_{1,2,3,5}$ is zero then in general the coefficients of $x_{3}$ and $y_{3}$ are not both equal to 0 and hence our equation implies that $T_{1,3,4,5}$ is also equal to 0 .

Note that since the term $\mathbf{m}_{2}^{T} \mathbf{F}_{1,2} \mathbf{m}_{1}$ is multiplied by three factors, namely $x_{3}, y_{2}$, and $\left(\mathbf{F}_{1,3} \mathbf{m}_{1}\right)_{3}$, this situation may appear in other cases where those factors become 0 . In the case where $x_{3}=0$, it is easily verified that the two trilinearities are not algebraically dependent since, for example, $T_{1,2,3,5}=0$ and $x_{3}=0$ imply that $\operatorname{coeff}\left(T_{1,2,3,5}, y_{3}\right)=0$. On the other hand the two cases $y_{2}=0$ and $\left(\mathbf{F}_{1,3} \mathbf{m}_{1}\right)_{3}$ do imply that the two trilinearities are algebraically dependent.

Let us now study in more detail the information contained in the trilinearities. 


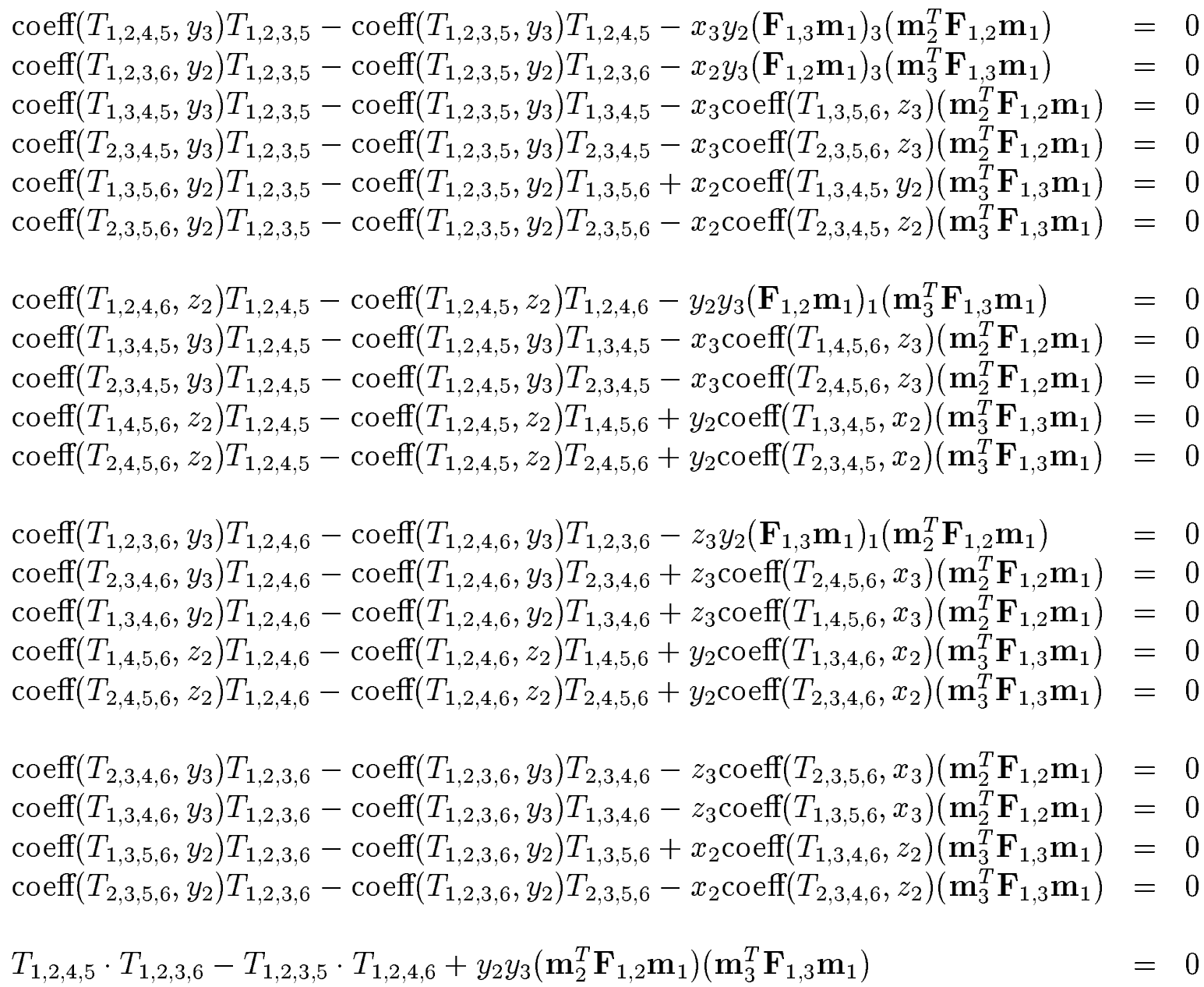

Table 2: The relations of the elements in the group I. 


\subsection{Geometric Information contained in the trilineari- ties}

We have seen that if three image points $m_{1}, m_{2}, m_{3}$ are such that they zero a given trilinearity, for example $T_{1,2,3,5}$, it means that the optical ray of $m_{1}$ intersects the line of intersection of two planes going through the optical ray of $m_{2}$ and $m_{3}$, respectively (see figure 1 ).

\subsection{1 $\left(m_{1}, m_{2}, m_{3}\right)$ zero two trilinear relations}

Let us now see what happens if the three points are such that they zero another trilinearity. Several cases are possible: first the second trilinearity can be in the same group, i.e I, or not, and second it can be related to $T_{1,2,3,5}$ or not. Of course, the geometric information contained in the fact that two trilinearities are equal to 0 is equivalent to the one contained in their algebraic relations.

Let us consider the case of $T_{1,2,4,5}$, also in group I, which is related to $T_{1,2,3,5}$. The optical rays of $m_{1}$ and $m_{2}$ intersect which can also be understood algebraically by the fact that the two trilinearities are related through the fundamental matrix $\mathbf{F}_{1,2}$, as shown in table 2.

Let us now move to the case of $T_{1,2,4,6}$, also in the group I, but unrelated to $T_{1,2,3,5}$. The situation is shown in figure 3 : the optical ray of $m_{1}$ intersects the two lines of intersection of the planes $x_{2} 5-y_{2} 4$ and $x_{3} 8-y_{3} 7$ on one hand and $y_{2} \mathbf{6}-z_{2} \mathbf{5}$ and $y_{3} \mathbf{9}-z_{3} \mathbf{8}$ on the other hand. In particular, it is seen in this figure that none of the three optical rays intersect in general. On the other hand, if we assume that any two of the three optical rays intersect, then it implies generically that the third optical ray goes through their point of intersection.

Let us now move to the case of $T_{1,3,4,5}$, in the group II, and related to $T_{1,2,3,5}$ through the fundamental matrix $\mathbf{F}_{1,2}$. The optical rays of $m_{1}$ and $m_{2}$ intersect which can also be understood algebraically by the fact that the two trilinearities are related through the fundamental matrix $\mathbf{F}_{1,2}$, as shown in table 2 .

Let us finally consider the case of $T_{2,3,4,6}$, in the group II, but unrelated to $T_{1,2,3,5}$. Like in the second case above, the fact that $T_{1,2,3,5}$ and $T_{2,3,4,6}$ are both equal to 0 does not imply that the three optical rays intersect at a point. 


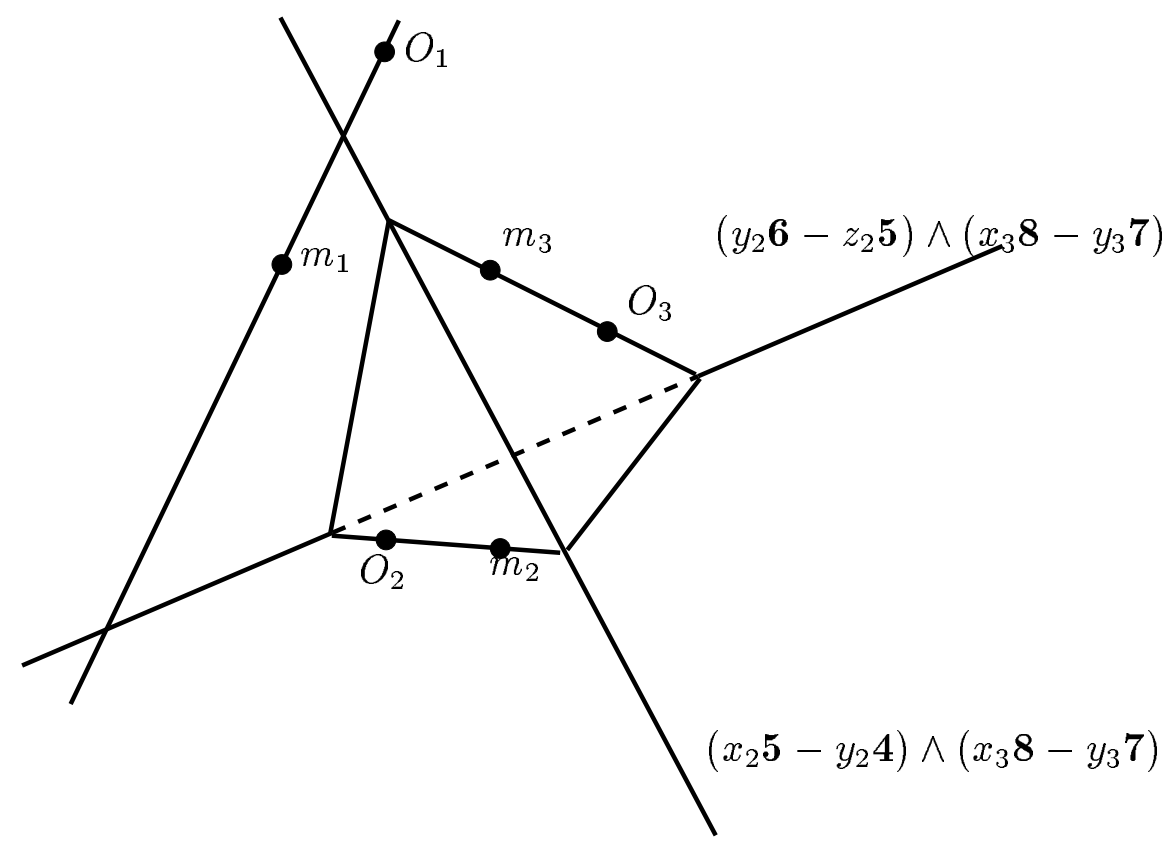

Figure 3: A geometric interpretation of $T_{1,2,3,5}$ and $T_{1,2,4,6}$ : none of the three optical rays intersect in general.

$\operatorname{RR} \mathrm{n}^{\circ} 2665$ 


\subsection{2 $\left(m_{1}, m_{2}, m_{3}\right)$ zero three or more trilinear relations}

From table 2, we can extend this kind of analysis to the case where more than two trilinearities are equal to 0 . One of the salient results of this analysis is that if four trilinearities within the same group are equal to 0 for some triplet of image points, this does not imply that the three corresponding optical rays intersect. For example, in the case of the group I, it only implies that the first optical ray intersects the second and the third. Similarly, for the group II, it only implies that the second optical ray intersects the first and the third and, for the group III, it only implies that the third optical ray intersects the first and the second.

In order for the three optical rays to intersect at a point, one trilinearity must be chosen from each group. For example, if we choose $T_{1,2,3,5}$ in the group I, $T_{1,3,4,5}$ in the group II, and $T_{1,3,5,6}$ in the group III, then it can be seen from table 2 that the conjunction of the first and the second trilinearities implies in general that the first two points satisfy the epipolar constraint, the conjunction of the second and the third trilinearities implies in general that the second and the third points satisfy the epipolar constraint (apply Cramer's rule to the rows $\left(\mathbf{C}_{1}, \mathbf{C}_{3}, \mathbf{C}_{4}, \mathbf{C}_{5}, \mathbf{C}_{6}\right)$ ), and that the conjunction of the first and the third trilinearities implies in general that the first and the third points also satisfy the epipolar constraint (see table 2). Furthermore, it can be seen that three is the smallest number of trilinearities necessary to ensure that the threee optical rays intersect.

\subsection{Recovering the three fundamental matrices from two trilinearities}

On the other hand, is it possible to recover the binary relations from a smaller number of trilinearities? This is interesting because this would be an alternative way to estimate the epipolar geometry of a set of three cameras. We now show that the complete epipolar geometry or, equivalently the three fundamental matrices, can be recovered from the knowledge of only two of the trilinearities. 


\subsubsection{Recovering the epipoles}

Let us start with the epipoles. It is clear geometrically and can be verified algebraically that for each of the twelve trilinearities, let us call it $T$ for simplicity, the following three relations hold:

$$
\begin{aligned}
& T\left(m_{1}, e_{2,1}, e_{3,1}\right)=0 \forall m_{1} \\
& T\left(e_{1,2}, m_{2}, e_{3,2}\right)=0 \forall m_{2} \\
& T\left(e_{1,3}, e_{2,3}, m_{3}\right)=0 \forall m_{3}
\end{aligned}
$$

Let us consider in turn each one these three relations and show how they can be used to determine effectively the epipoles. In the case of $T_{1,2,3,5}$, we rewrite it as

$$
T_{1,2,3,5}\left(m_{1}, m_{2}, m_{3}\right)=A\left(m_{2}, m_{3}\right) x_{1}+B\left(m_{2}, m_{3}\right) y_{1}+C\left(m_{2}, m_{3}\right) z_{1}
$$

Each polynomial $A, B, C$ is homogeneous linear in the coordinates $x_{2}, y_{2}$ and $x_{3}, y_{3}$. Hence each one of them defines a collineation from $\mathcal{P}^{1}$ to $\mathcal{P}^{1}$. Let us call these collineations $F_{A}, F_{B}, F_{C}$. In order to see this, let us for example write

$$
A\left(m_{2}, m_{3}\right)=c x_{2} x_{3}+d x_{2} y_{3}-a y_{2} x_{3}-b y_{2} y_{3}
$$

the condition $A\left(m_{2}, m_{3}\right) \equiv 0$ is equivalent to

$$
\theta_{2}=\frac{a \theta_{3}+b}{c \theta_{3}+d}
$$

where we have defined $\theta_{2}=\frac{x_{2}}{y_{2}}$ and $\theta_{3}=\frac{x_{3}}{y_{3}}$ as two projective parameters on the two projective lines. Because $T\left(m_{1}, e_{2,1}, e_{3,1}\right)=0 \forall m_{1}$ implies that $A\left(e_{2,1}, e_{3,1}\right)=B\left(e_{2,1}, e_{3,1}\right)=C\left(e_{2,1}, e_{3,1}\right)=0$, we know that there exists at least one value $\theta_{2}^{0}$ of $\theta_{2}$ such that:

$$
F_{A}\left(\theta_{2}^{0}\right)=F_{B}\left(\theta_{2}^{0}\right)=F_{C}\left(\theta_{2}^{0}\right)
$$

This is equivalent to the fact that $\theta_{2}^{0}$ is a fixed point of the two collineations $F_{A}^{-1} F_{B}$ and $F_{B}^{-1} F_{C}$. The fixed points of these two collineations are obtained by solving the two quadratic equations

$$
\theta=F_{A}^{-1} F_{B}(\theta) \text { and } \theta=F_{B}^{-1} F_{C}(\theta)
$$

$\operatorname{RR} \mathrm{n}^{\circ} 2665$ 
The coefficients of these two equations are shown to be of degree two in the brackets and it is shown by reduction and normalization that they are both divisable by $e_{2,1}[2] \theta_{2}-e_{2,1}[1]$, the remaining factors being in general different. This shows constructively how to recover the ratio of the first two coordinates of $e_{2,1}$ from $T_{1,2,3,5}$. Of course, the ratio of the first two coordinates of $e_{3,1}$ is obtained, for example, from the one of those of $e_{2,1}$ by applying any one of the three collineations. The same reasoning applied to $T_{1,2,4,6}$ shows how to recover the ratio of the second to the third coordinates of $e_{2,1}$ and $e_{3,1}$.

Let us now consider the second of the three equations (10) and show that the coordinates of $e_{1,2}, e_{1,3}, e_{3,2}$, and $e_{3,1}$ can be recovered from $T_{1,2,3,5}$ and $T_{1,2,4,6}$. Let us rewrite the first trilinearity as follows

$$
T_{1,2,3,5}=P\left(m_{1}, m_{3}\right) x_{2}+Q\left(m_{1}, m_{3}\right) y_{2}
$$

the coefficients $P$ and $Q$ are homogeneous and linear in the coordinates of $m_{1}$ and in the first two coordinates of $m_{3}$. This means that they can be rewritten as

$$
P\left(m_{1}, m_{3}\right)=l\left(m_{1}\right) x_{3}+l_{1}\left(m_{1}\right) y_{3} \text { and } Q\left(m_{1}, m_{3}\right)=l_{2}\left(m_{1}\right) x_{3}+l_{3}\left(m_{1}\right) y_{3}
$$

The polynomial $l\left(m_{1}\right)\left(\right.$ resp. $\left.l_{1}\left(m_{1}\right), l_{2}\left(m_{1}\right), l_{3}\left(m_{1}\right)\right)$ is homogeneous and linear in the coordinates of $m_{1}$ and hence defines a line in the first retinal plane. The equation $P\left(m_{1}, m_{3}\right)=0\left(\right.$ resp. $\left.Q\left(m_{1}, m_{3}\right)=0\right)$ therefore defines a pencil of lines generated by $l$ and $l_{1}$ (resp. $l_{2}$ and $l_{3}$ ) in the first retinal plane. Since these two pencils have the same projective parameter $\left(x_{3}, y_{3}\right)$, they are in projective correspondence. It is known that in that case (see for example [24]), the points of intersection of two corresponding lines of each pencil is on a conic. In our case, the equation of that conic is very simple :

$$
l\left(m_{1}\right) l_{3}\left(m_{1}\right)-l_{1}\left(m_{1}\right) l_{2}\left(m_{1}\right)=0
$$

Let us show that this conic is singular and made of two lines which are the images in the first retina of the lines $4 \wedge \mathbf{5}$ and $\mathbf{7} \wedge \mathbf{8}$, going through the second and the third optical centers, respectively. In order to see this, let us consider first the line $4 \wedge \mathbf{5}$. Any optical ray $\left\langle O_{1}, m_{1}\right\rangle$ that intersects this line at a point $M$ defines a plane going through this point and the line $7 \wedge 8$. It is clear geometrically that for all planes going through the line $\mathbf{4} \wedge \mathbf{5}$ (i.e. for all 
$x_{2}, y_{2}$ in the equation of $\left.T_{1,2,3,5}\right)$ the line of intersection of that plane with the previous plane through $7 \wedge 8$ and $M$ will go through $M$ and hence intersect $\left\langle O_{1}, m_{1}\right\rangle$ (see figure 4 ). The conic therefore contains the image through the first camera of the line $4 \wedge \mathbf{5}$.

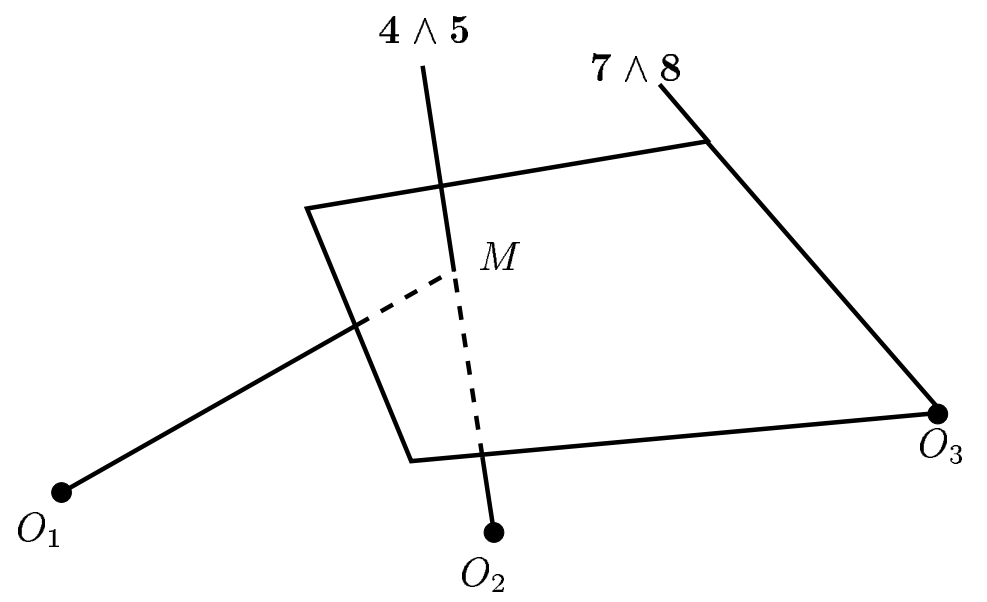

Figure 4: The conic contains the image of the line $4 \wedge \mathbf{5}$.

Let us now consider the line $7 \wedge 8$ and the plane defined by this line and the optical center $O_{1}$. It is $\mathbf{7} \wedge \mathbf{8}$. For each plane going through the line $\mathbf{4} \wedge \mathbf{5}$ (i.e. for all $x_{2}, y_{2}$ ) the line of intersection of that plane with the previous plane is of course contained in that plane and hence intersects all optical rays with origin $O_{1}$ and contained in the plane defined by $O_{1}$ and $7 \wedge 8$ (see figure 5). The conic therefore contains the image through the first camera of the line $7 \wedge 8$.

The corresponding situation in the first retinal is shown in figure 6 . We have represented in this figure the lines of equation $l=0$ (resp. $\left.l_{2}=0\right)$, which is the image of the line of the intersection, noted $5 \wedge 8$ (resp. $4 \wedge 8$ ), of the planes 5 and 8 (resp. 4 and 8 ), the lines of equation $l_{1}=0\left(\right.$ resp. $\left.l_{3}=0\right)$, which is the image of the line of the intersection, noted $5 \wedge 7($ resp. $4 \wedge 7)$, of the planes 5 and 7 (resp. 4 and 7 ), of the two pencils, as well as the lines, noted $\mathbf{4} \wedge \mathbf{5}$ and $\mathbf{7} \wedge \mathbf{8}$, which form the conic oocus of the points of intersection of the corresponding lines of the two pencils.

$\operatorname{RR} \mathrm{n}^{\circ} 2665$ 


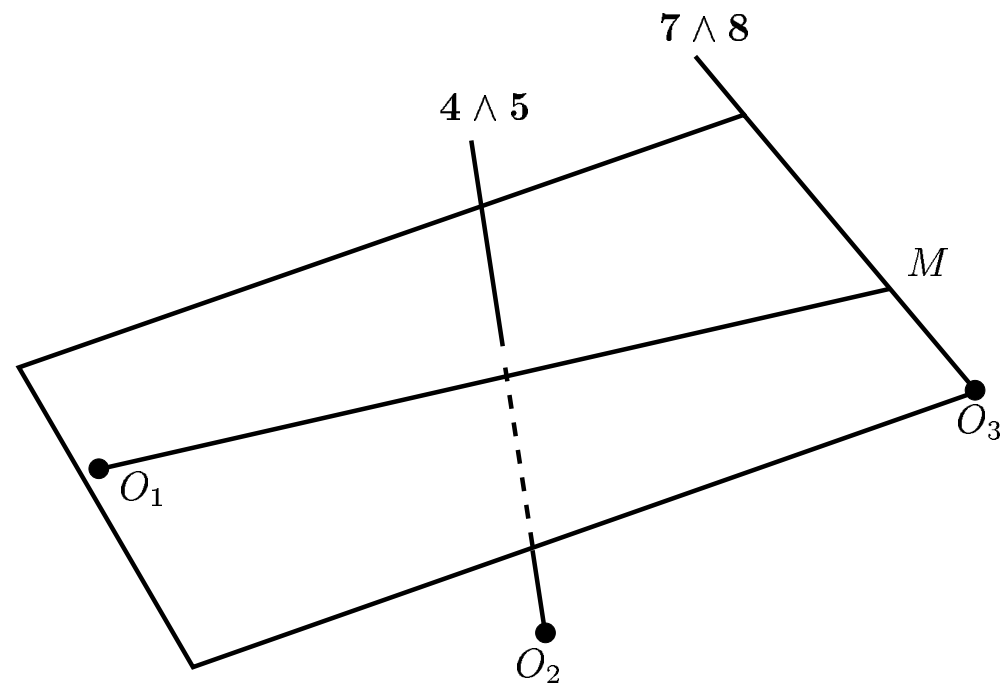

Figure 5: The conic contains the image of the line $7 \wedge 8$.

Note that the epipole $e_{1,2}$ is on the image of the line $\mathbf{4} \wedge \mathbf{5}$ and the epipole $e_{1,3}$ on the image of the line $\mathbf{7} \wedge \mathbf{8}$. These two lines are obtained in a straightforward fashion from the trilinearity $T_{1,2,3,5}$.

If we now consider the trilinearity $T_{1,2,4,6}$, we obtain in a similar fashion the image of the line $\mathbf{8} \wedge \mathbf{9}$ as the line joining the vertices of two pencils of lines and the image of the line $\mathbf{5} \wedge \mathbf{6}$ as the line $4 \wedge \mathbf{5}$ in the previous case. The epipoles $e_{1,2}$ and $e_{1,3}$ are obtained by intersecting the images of the lines $\mathbf{4} \wedge \mathbf{5}$ and $\mathbf{5} \wedge \mathbf{6}$, and $\mathbf{7} \wedge \mathbf{8}$ and $\mathbf{8} \wedge \mathbf{9}$, respectively. For each epipole, we also recover the corresponding epipole in the third image, namely $e_{3,2}$ for $e_{1,2}$ and $e_{3,1}$ for $e_{1,3}$. Indeed, the point $e_{1,2}$ is on the image of the line $\mathbf{4} \wedge \mathbf{5}$ and corresponds to a unique value of the ratio of the coordinates $x_{3}$ and $y_{3}$ when one considers the two pencils of lines obtained from $T_{1,2,3,5}$ and to a unique value of the ratio of the coordinates $y_{3}$ and $z_{3}$ when one considers the two pencils of lines obtained from $T_{1,2,4,6}$. This yields the coordinates of $e_{3,2}$. A similar reasoning applies for $e_{3,1}$. 


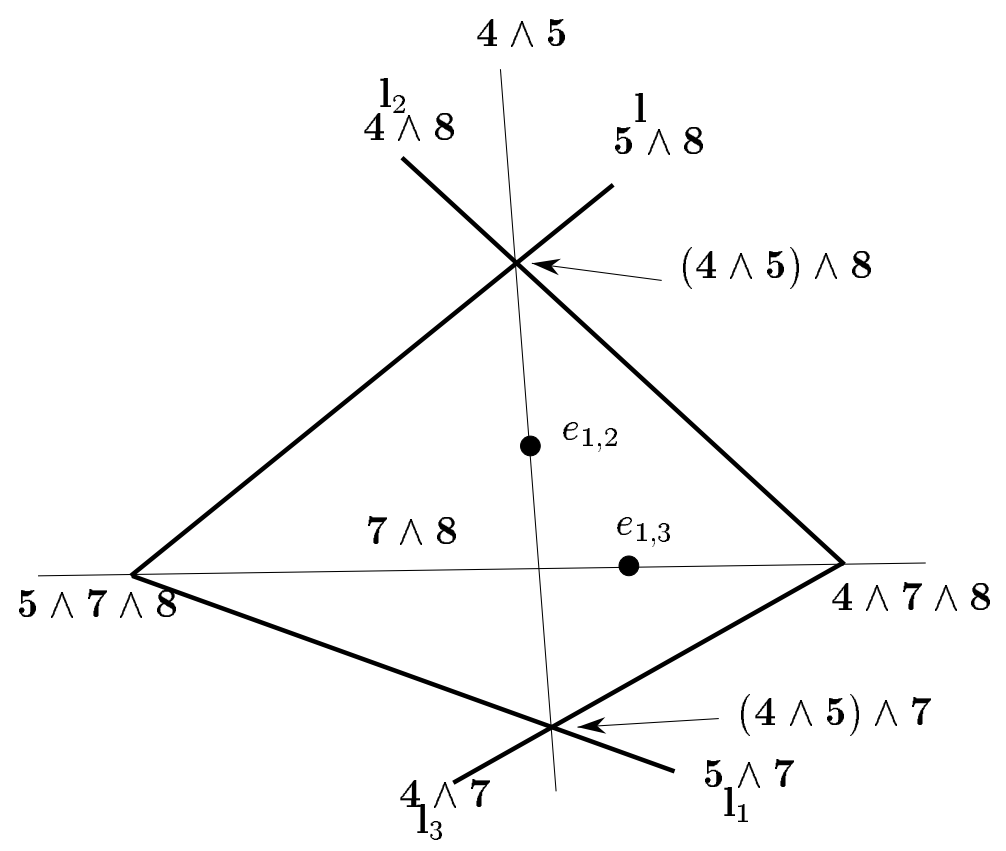

Figure 6: The locus of the points of intersection of corresponding lines in the two pencils is decomposed into two lines (see text). 
We still have to determine $e_{2,3}$. This can be done using the third equation (10), exchanging the roles of $m_{2}$ and $m_{3}$, and writing:

$$
T_{1,2,3,5}=R\left(m_{1}, m_{2}\right) x_{3}+S\left(m_{1}, m_{2}\right) y_{3}
$$

the coefficients $R$ and $S$ are homogeneous and linear in the coordinates of $m_{1}$ and in the first two coordinates of $m_{2}$. They can be rewritten as

$$
R\left(m_{1}, m_{2}\right)=l\left(m_{1}\right) x_{2}+l_{2}\left(m_{1}\right) y_{2} \text { and } S\left(m_{1}, m_{2}\right)=l_{1}\left(m_{1}\right) x_{2}+l_{3}\left(m_{1}\right) y_{2}
$$

Like in the previous case, each equation $R\left(m_{1}, m_{2}\right)=0$ and $S\left(m_{1}, m_{2}\right)=0$ defines a pencil of lines in the first retinal plane. Since these two pencils have the same projective parameter $\left(x_{2}, y_{2}\right)$, they are in projective correspondence. The locus of the points of intersection of two corresponding lines is the same conic as in the previous case which we know to be degenerate. Hence, the ratio of the first two coordinates of $e_{2,3}$ (resp. $e_{2,1}$ ) are obtained from the projective parameter corresponding to the lines of the pencils going through the point $e_{1,3}$ (resp. $e_{1,2}$ ). Just like before, the ratios of the second coordinates can be obtained from $T_{1,2,4,6}$.

\subsubsection{Recovering the fundamental matrices}

Now that we have recovered the epipoles, let us show how to recover completely the fundamental matrices. This can be seen first geometrically and then algebraically. Consider the points $\left(m_{1}, m_{2}\right)$ such that $T_{1,2,3,5}\left(m_{1}, m_{2}, e_{3,1}\right)=0$. Since $e_{3,1}$ is the image in the third retina of $O_{1}$, this means that for each point $m_{3}$ of the plane $\pi$ defined by $O_{1}$ and $7 \wedge \mathbf{8}$ and each pair $\left(m_{1}, m_{2}\right)$ such that $T_{1,2,3,5}\left(m_{1}, m_{2}, e_{3,1}\right)=0$ we have $T_{1,2,3,5}\left(m_{1}, m_{2}, m_{3}\right)=0$. Because of the geometric interpretation of this relation, this implies that the optical rays $m_{1}$ and $m_{3}$ intersect, even though the point $m_{3}$ is left unspecified in the plane $\pi$. Therefore, there must be some information about $\mathbf{F}_{1,3}$ within $T_{1,2,3,5}\left(m_{1}, m_{2}, e_{3,1}\right)$. This can be checked algebraically since, after reduction, we find that

$$
T_{1,2,3,5}\left(m_{1}, m_{2}, e_{3,1}\right)=\left(\mathbf{F}_{1,3} \mathbf{m}_{1}\right)_{3}\left(x_{2} \mathbf{e}_{2,1}[2]-y_{2} \mathbf{e}_{2,1}[1]\right)
$$

Since we now know the epipoles, this yields the third row of $\mathbf{F}_{1,3}$. Similarly, if we consider the relation $T_{1,2,4,6}\left(m_{1}, m_{2}, e_{3,1}\right)=0$, we obtain

$$
T_{1,2,4,6}\left(m_{1}, m_{2}, e_{3,1}\right)=\left(\mathbf{F}_{1,3} \mathbf{m}_{1}\right)_{1}\left(y_{2} \mathbf{e}_{2,1}[3]-z_{2} \mathbf{e}_{2,1}[2]\right)
$$


which yields the first row of $\mathbf{F}_{1,3}$. Having two rows and the two epipoles, it is easy to compute the third row to completely determine the fundamental matrix $\mathbf{F}_{1,3}$.

In a similar fashion, the expressions $T_{1,2,3,5}\left(m_{1}, e_{2,1}, m_{3}\right)$ and $T_{1,2,4,6}\left(m_{1}, e_{2,1}, m_{3}\right)$ can be used to determine the fundamental matrix $\mathbf{F}_{1,2}$.

In order to determine $\mathbf{F}_{2,3}$, we can proceed as follows. Consider a point $m_{2}$ and its epipolar line in the first image, represented by $\mathbf{F}_{2,1} \mathbf{m}_{2}$. Choose any point $m_{1}$ different from $e_{1,2}$ on that line and use the two trilinearities $T_{1,2,3,5}$ and $T_{1,2,4,6}$ to predict $m_{3}$ in the third image. Since by construction $m_{1}$ and $m_{2}$ satisfy the epipolar constraint, we know from section 4.2 that the three optical rays $\left\langle O_{i}, m_{i}\right\rangle, i=1,2,3$ intersect at a point. Hence the point $m_{3}$ must be on the epipolar line of $m_{2}$ in the third retinal plane, represented by $\mathbf{F}_{2,3} \mathbf{m}_{2}$. When $m_{1}$ moves along the previous epipolar line, $m_{3}$ moves on a locus which contains the epipolar line of $m_{2}$. Since the coordinates of the predicted point $m_{3}$ are quadratic functions of the coordinates of $m_{1}$, its locus is a conic which can be shown by some simple algebraic manipulations to split into two lines, the line of equation $y_{3}=0$ and the line of equation $\mathbf{m}_{3}^{T} \mathbf{F}_{2,3} \mathbf{m}_{2}$. In detail, the equation of the locus is:

$$
\left(y_{2} e_{2,1}[3]-z_{2} e_{2,1}[2]\right)\left(y_{2} e_{2,1}[1]-x_{2} e_{2,1}[2]\right) e_{1,2}[3] y_{3} \mathbf{m}_{3}^{T} \mathbf{F}_{2,3} \mathbf{m}_{2}=0
$$

This completes the determination of the epipolar geometry of the three cameras from two of the trilinearities in group I, $T_{1,2,3,5}$ and $T_{1,2,4,6}$.

\subsection{Algebraic constraints on the coefficients of the tri- linearities within the same group}

It is straightforward to show that each group of four trilinearities depends upon 27 projective parameters. We have also shown that those trilinearities can be used to recover the three fundamental matrices and ffrom which it is known that the three perspective projection matrices of the three cameras can be recovered up to an unknown collineation of 3-space. It is also known that the number of degrees of freedom is equal to 18 . There must therefore exist 8 constraints that are satisfied by the 27 free parameters. We are now going to discover these constraints using the previous study.

$\operatorname{RR} \mathrm{n}^{\circ} 2665$ 
To fix the ideas, let us work with $T_{1,2,3,5}$. With the same notations as in section 4.3.1, we write it as

$$
T_{1,2,3,5}\left(m_{1}, m_{2}, m_{3}\right)=P\left(m_{1}, m_{3}\right) x_{2}+Q\left(m_{1}, m_{3}\right) y_{2}=R\left(m_{1}, m_{2}\right) x_{3}+S\left(m_{1}, m_{2}\right) y_{3},
$$

and introduce the four lines $l, l_{1}, l_{2}$, and $l_{3}$ of the first retinal plane which are the images of the 3 -D lines $5 \wedge \mathbf{8}, \mathbf{5} \wedge \mathbf{7}, \mathbf{4} \wedge \mathbf{7}$ and $\mathbf{4} \wedge \mathbf{8}$, respectively. The conditions that must be satisfied by the coefficients $a, b, c$ and $a_{i}, b_{i}, c_{i}, i=1, \cdots, 3$ are obtained from the study in the previous section.

First, writing that the three collineations $F_{A}, F_{B}$ and $F_{C}$ satisfy the condition (11), we can eliminate $\theta_{0}^{2}$ and obtain a polynomial condition of degree 8 in the coefficients $a, b, c$ and $a_{i}, b_{i}, c_{i}, i=1, \cdots, 3$. Let us denote it by $H$.

Second, we considered earlier the two pencils of lines

$$
\begin{gathered}
x_{3} l+y_{3} l_{1} \\
x_{3} l_{2}+y_{3} l_{3}
\end{gathered},
$$

and

$$
\begin{gathered}
x_{2} \mathbf{l}+y_{2} \mathbf{l}_{2} \\
x_{2} \mathbf{l}_{1}+y_{2} \mathbf{l}_{3}
\end{gathered}
$$

and we saw that the points of intersection of the corresponding lines of these two pencils had to lie on two degenerate conics. In order for this to be true, it is necessary and sufficient that the value of $x_{3}, y_{3}$ (resp. of $x_{2}, y_{2}$ ) for which the corresponding line of the first pencil goes through the vertex of the second pencil is the same as the value of $x_{3}, y_{3}$ (resp. of $x_{2}, y_{2}$ ) for which the corresponding line of the second pencil goes through the vertex of the first one. In that case, the conics degenerate in the line going through the two vertexes and the line going through the two points of intersection of the lines corresponding to the value 0 of $x_{3}$ (resp. of $x_{2}$ ) and to the value 0 of $y_{3}$ (resp. $y_{2}$ ).

Writing these conditions, we obtain two polynomials of degree six in the parameters $a_{i}, b_{i}, c_{i}, i=1, \cdots, 4$ which are identical. Let us denote it by $K$. It is found furthermore that the following relation between $H$ and $K$ is true

$$
H=K\left(b_{3} b_{2}-b_{4} b_{1}\right)
$$

which shows that $H$ is in the ideal generated by $K$ or, simpler, that there is only one necessary condition on the parameters $a, b, c$ and $a_{i}, b_{i}, c_{i}, i=1, \cdots, 3$, 
namely $K=0$. It can be easily shown that $K$ is a polynomial of degree 2 in some $3 \times 3$ determinants involving the vectors $1,1_{i}, i=1, \cdots, 3$ :

$$
K \equiv\left|\begin{array}{lll}
1 & l_{2} & l_{3}
\end{array}\right|\left|\begin{array}{lll}
1 & l_{1} & l_{3}
\end{array}\right|-\left|\begin{array}{lll}
l_{1} & l_{2} & l_{3}
\end{array}\right|\left|\begin{array}{lll}
1 & l_{1} & l_{2}
\end{array}\right|=0
$$

We find it convenient now to move our analysis to $T_{1,2,4,6}$. It shares only three of its coefficients with $T_{1,2,3,5}$, those of 1 . Therefore we introduce three new lines $\mathbf{l}_{1}^{\prime}=\left[a_{1}^{\prime}, b_{1}^{\prime}, c_{1}^{\prime}\right]^{T}, \mathbf{l}_{2}^{\prime}=\left[a_{2}^{\prime}, b_{2}^{\prime}, c_{2}^{\prime}\right]^{T}$ and $\mathbf{l}_{3}^{\prime}=\left[a_{3}^{\prime}, b_{3}^{\prime}, c_{3}^{\prime}\right]^{T}$ and write $T_{1,2,4,6}$ as

$$
\begin{aligned}
& T_{1,2,4,6}=y_{2}\left(y_{3}\left(a_{1}^{\prime} x_{1}+b_{1}^{\prime} y_{1}+c_{1}^{\prime} z_{1}\right)+z_{3}\left(a_{2}^{\prime} x_{1}+b_{2}^{\prime} y_{1}+c_{2}^{\prime} z_{1}\right)\right)+ \\
& \quad+z_{2}\left(y_{3}\left(a_{3}^{\prime} x_{1}+b_{3}^{\prime} y_{1}+c_{3}^{\prime} z_{1}\right)+z_{3}\left(a x_{1}+b y_{1}+c z_{1}\right)\right)
\end{aligned}
$$

A similar reasoning as the one conducted for $T_{1,2,3,5}$ shows that these coefficients have to satisfy the condition

$$
\left|\begin{array}{lll}
1 & l_{1}^{\prime} & l_{3}^{\prime}
\end{array}\right|\left|\begin{array}{lll}
1 & l_{1}^{\prime} & l_{2}^{\prime}
\end{array}\right|-\left|\begin{array}{lll}
1 & l_{2}^{\prime} & l_{3}^{\prime}
\end{array}\right|\left|\begin{array}{lll}
l_{1}^{\prime} & l_{2}^{\prime} & l_{3}^{\prime}
\end{array}\right|=0
$$

Moving now to $T_{1,2,4,5}$, it is seen that only three new coefficients appear, as compared to $T_{1,2,3,5}$ and $T_{1,2,4,6}$. Therefore we introduce one more line $\mathbf{l}_{2}^{\prime \prime}$ and write $T_{1,2,4,5}$ as

$$
\begin{aligned}
& T_{1,2,4,5}=y_{2}\left(x_{3}\left(a_{2}^{\prime} x_{1}+b_{2}^{\prime} y_{1}+c_{2}^{\prime} z_{1}\right)+y_{3}\left(a_{2}^{\prime \prime} x_{1}+b_{2}^{\prime \prime} y_{1}+c_{2}^{\prime \prime} z_{1}\right)\right)- \\
& \quad+z_{2}\left(x_{3}\left(a x_{1}+b y_{1}+c z_{1}\right)+y_{3}\left(a_{1} x_{1}+b_{1} y_{1}+c_{1} z_{1}\right)\right)
\end{aligned}
$$

The same reasoning as before shows that these coefficients must satisfy the condition

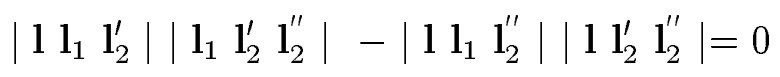

Finally we move to $T_{1,2,3,6}$ for which it is only necessary to add a ninth line, noted $\mathbf{l}_{3}^{\prime \prime}$ :

$$
\begin{aligned}
& T_{1,2,3,6}=-x_{2}\left(y_{3}\left(a_{3}^{\prime} x_{1}+b_{3}^{\prime} y_{1}+c_{3}^{\prime} z_{1}\right)+z_{3}\left(a x_{1}+b y_{1}+c z_{1}\right)\right)+ \\
& \quad+y_{2}\left(y_{3}\left(a_{3}^{\prime \prime} x_{1}+b_{3}^{\prime \prime} y_{1}+c_{3}^{\prime \prime} z_{1}\right)-z_{3}\left(a_{2} x_{1}+b_{2} y_{1}+c_{2} z_{1}\right)\right)
\end{aligned}
$$

And we find the constraint

$$
\left|\begin{array}{lll}
l_{2} & l_{3}^{\prime} & l_{3}^{\prime \prime}
\end{array}\right|\left|\begin{array}{lll}
1 & l_{2} & l_{3}^{\prime}
\end{array}\right|+\left|\begin{array}{lll}
1 & l_{2} & l_{3}^{\prime \prime}
\end{array}\right|\left|\begin{array}{lll}
1 & l_{3}^{\prime} & l_{3}^{\prime \prime}
\end{array}\right|=0
$$

$\operatorname{RR} \mathrm{n}^{\circ} 2665$ 
Four further constraints must be satisfied by the 27 coefficients of the four trilinearities in the same group. They can be seen as consistency constraints so that the estimates of the epipolar geometry provided by $T_{1,2,4,5}$ and $T_{1,2,3,6}$ are consistent with those provided by $T_{1,2,3,5}$ and $T_{1,2,4,6}$.

For example, the line $\mathbf{l}_{2}^{\prime \prime}$ must satisfy two other conditions than (14). The first condition can be obtained by noticing that the point of intersection of $l_{2}^{\prime \prime}$ and $l_{1}(5 \wedge 6 \wedge 7)$ must be the same as that of $l_{1}$ and the line going through the points $1 \wedge l_{2}^{\prime}$ and $l_{1}^{\prime} \wedge l_{3}^{\prime}$. The second condition is that the point of intersection of $\mathrm{l}_{2}^{\prime \prime}$ and $\mathrm{l}_{2}^{\prime}(6 \wedge 7 \wedge 8)$ must be the same as that of $\mathrm{l}_{2}^{\prime}$ and the line going through the points $1 \wedge l_{1}$ and $l_{2} \wedge l_{3}$.

Similarly, for $T_{1,2,3,6}$, it can be seen that the lines $\mathbf{l}_{3}^{\prime \prime}, \mathbf{l}_{3}^{\prime}$ and $\left(1 \wedge \mathbf{l}_{2}\right) \wedge\left(\mathbf{l}_{1} \wedge \mathbf{l}_{3}\right)$ must go through a common point or that the lines $\mathbf{l}_{3}^{\prime \prime}, \mathbf{l}_{2}$ and $\left(\mathbf{l}_{1}^{\prime} \wedge \mathbf{l}_{2}^{\prime}\right) \wedge\left(1 \wedge \mathbf{l}_{3}^{\prime}\right)$ must go through a common point.

Another way to state this result is to say that once $T_{1,2,3,5}$ and $T_{1,2,4,6}$ are defined and satisfy (12) and (13), $\mathbf{l}_{2}^{\prime \prime}$ (resp. $\mathbf{l}_{3}^{\prime \prime}$ ) is completely determined: it goes through the points $A_{2}$ and $B_{2}$ (resp. $A_{3}$ and $B_{3}$ ) defined as follows: $A_{2}$ (resp. $\left.A_{3}\right)$ is the point of intersection of $\mathrm{l}_{1}$ (resp. $\mathrm{l}_{2}$ ) and the line going through the points $1 \wedge \mathbf{l}_{2}^{\prime}$ and $\mathbf{l}_{1}^{\prime} \wedge \mathrm{l}_{3}^{\prime}$ (resp. through the points $\mathbf{l} \wedge \mathrm{l}_{3}^{\prime}$ and $\left.\mathbf{l}_{1}^{\prime} \wedge \mathrm{l}_{2}^{\prime}\right) ; B_{2}$ (resp. $\left.B_{3}\right)$ is the point of intersection of $\mathbf{l}_{2}^{\prime}\left(\right.$ resp. $\mathbf{l}_{3}^{\prime}$ ) and the line going through $1 \wedge \mathrm{l}_{1}$ $\left(\right.$ resp. $\left.1 \wedge l_{2}\right)$ and $l_{2} \wedge l_{3}$ (resp. $\left.l_{1} \wedge l_{3}\right)$. This does not completely determine $l_{2}^{\prime \prime}$ and $l_{3}^{\prime \prime}$, since they still depend upon a scale factor which is fixed by the two further conditions (14) and (15). This is shown in figure 7.

Note that as a byproduct of our analysis, we have obtained a minimal parameterization of the set of the twelve trilinearities with eighteen parameters: 27 parameters defined up to a scale factor minus our eight constraints, that is 18. A minimal parameterization is relatively easy to obtain as shown now.

Let us assume, for example, that the three vectors $\mathrm{l}_{i}, i=1,2,3$ are linearly independent and express 1 in the basis they form:

$$
\mathbf{l}=\sum_{i=1}^{3} \alpha_{i} \mathbf{l}_{i}
$$

Equation (12) then implies that

$$
\alpha_{1} \alpha_{2}+\alpha_{3}=0
$$




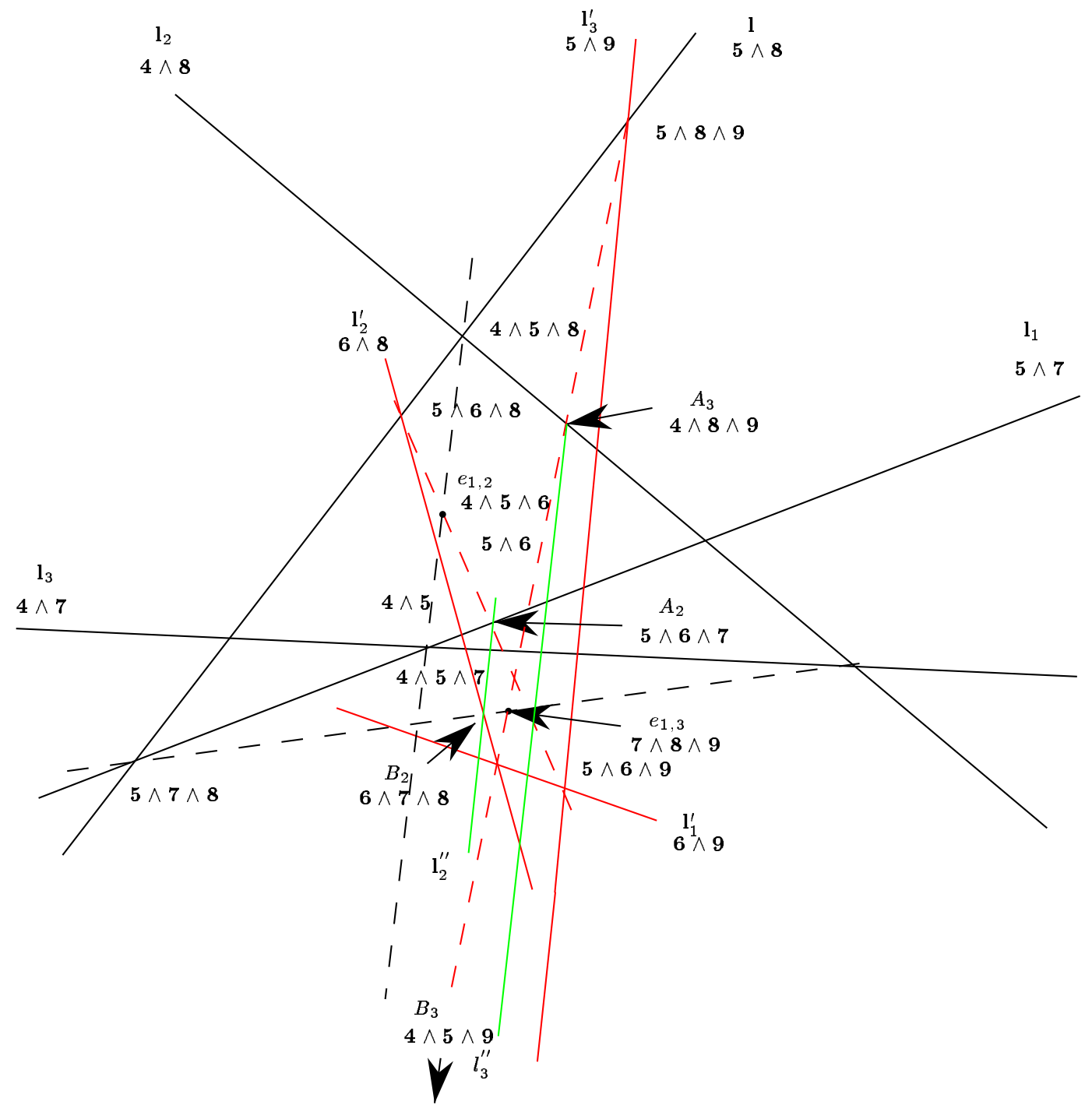

Figure 7: Determination of the lines $\mathbf{l}_{2}^{\prime \prime}$ and $\mathbf{l}_{3}^{\prime \prime}$. 
We are now using 11 parameters to represent $T_{1,2,3,5}$ : the 9 coordinates of the three vectors $\mathrm{l}_{i}, i=1,2,3, \alpha_{1}$ and $\alpha_{2}$. Moving on to $T_{1,2,4,6}$, we can use the 6 coordinates of, say, $\mathbf{l}_{1}^{\prime}$ and $\mathbf{l}_{2}^{\prime}$ and the first two coordinates $\beta_{i}, i=1,2,3$ of $l_{3}^{\prime}$ in the basis formed by $1, l_{1}^{\prime}, l_{2}^{\prime}$ since $\beta_{3}$ is obtained, because of (13), by the following equation:

$$
\beta_{1} \beta_{2}+\beta_{3}=0
$$

$l_{2}^{\prime \prime}$ and $l_{3}^{\prime \prime}$ are then obtained, as described previously, as homogeneous functions of the 19 coefficients that we have so far. Hence, all the coefficients of the trilinearities in the group I can be written as functions of those 19 parameters. Since we can apply a global scale factor to those coefficients, we have in fact a minimal representation with 18 parameters.

\subsection{Algebraic relations between the coefficients of the trilinearities in different groups}

There exist very simple relations between the coefficients of the trilinearities in the different groups. More specifically, the coefficients of each trilinearity in one group can be expressed as quadratic functions of the coefficients of at most two trilinearities in another group. For example, the coefficients of $T_{1,3,4,5}$ can be expressed as quadratic functions of those of $T_{1,2,3,5}$ and $T_{1,2,4,5}$ as shown now.

The key is to use once again Cramer's rule. Let us show as a start that the three coefficients of $x_{2}, y_{2}, z_{2}$ appearing in the coefficient of $x_{1} x_{3}$ can be expressed as a cross-product of two vectors of coefficients of $T_{1,2,3,5}$ and $T_{1,2,4,5}$. Indeed, let us apply Cramer's rule to the five vectors 4, 5, 6, 2 and 8 and multiply the resulting expression by $2 \wedge 8$. We obtain:

$$
[5,6,2,8] \mathbf{4} \wedge \mathbf{2} \wedge \mathbf{8}-[4,6,2,8] \mathbf{5} \wedge \mathbf{2} \wedge \mathbf{8}+[4,5,2,8] \mathbf{6} \wedge \mathbf{2} \wedge \mathbf{8}=\mathbf{0}
$$

Multiplying by 1 and rearranging the signs we obtain

$$
[2,4,5,8][1,2,6,8]+[2,5,6,8][1,2,4,8]+[2,6,4,8][1,2,5,8]=0
$$

Similarly, if we multiply by $\mathbf{3}$, we obtain

$$
[2,4,5,8][2,3,6,8]+[2,5,6,8][2,3,4,8]+[2,6,4,8][2,3,5,8]=0
$$


These two expressions show that the vector $\mathbf{c}_{28}$ of coordinates $[[2,4,5,8],[2,5,6,8],[2,6,4,8]]$ is proportional to the cross-product of the two vectors $\mathbf{c}_{128}=[[1,2,6,8],[1,2,4,8],[1,2,5,8]]$ and $\mathbf{c}_{238}=[[2,3,6,8],[2,3,4,8],[2,3,5,8]]$ whose coordinates appear in $T_{1,2,3,5}$ and $T_{1,2,4,5}$. More precisely, we have the following relation

$$
[1,2,3,8] \mathbf{c}_{28}=\mathbf{c}_{128} \times \mathbf{c}_{238}
$$

Note that the factor $[1,2,3,8]$ in front of $\mathbf{c}_{28}$ is the second coordinate of $e_{3,1}$.

If we now change 8 in 7 , we immediately obtain that the vector $\mathbf{c}_{27}=$ $[[2,4,5,7],[2,5,6,7],[2,6,4,7]]$ is proportional to the cross-product of the two vectors $\mathbf{c}_{127}=[[1,2,6,7],[1,2,4,7],[1,2,5,7]]$ and $\mathbf{c}_{237}=[[2,3,6,7],[2,3,4,7],[2,3,5,7]]$ whose coordinates also appear in $T_{1,2,3,5}$ and $T_{1,2,4,5}$. More precisely, we have the following relation

$$
[1,2,3,7] \mathbf{c}_{27}=\mathbf{c}_{127} \times \mathbf{c}_{237}
$$

Note that the factor $[1,2,3,7]$ in front of $\mathbf{c}_{27}$ is the first coordinate of $e_{3,1}$.

Let us now apply Cramer's rule to the five vectors $4,5,6,1$ and 8 and multiply the resulting expression by $\mathbf{1} \wedge \mathbf{8}$. We obtain:

$$
[5,6,1,8] \mathbf{4} \wedge \mathbf{1} \wedge \mathbf{8}-[4,6,1,8] \mathbf{5} \wedge \mathbf{1} \wedge \mathbf{8}+[4,5,1,8] \mathbf{6} \wedge \mathbf{1} \wedge \mathbf{8}=\mathbf{0}
$$

Multiplying by 2 and rearranging the signs we obtain

$$
[1,4,5,8][1,2,6,8]+[1,5,6,8][1,2,4,8]+[1,6,4,8][1,2,5,8]=0
$$

Similarly, if we multiply by $\mathbf{3}$, we obtain

$$
[1,4,5,8][3,1,6,8]+[1,5,6,8][3,1,4,8]+[1,6,4,8][3,1,5,8]=0
$$

These two expressions show that the vector $\mathbf{c}_{18}=[[1,4,5,8],[1,5,6,8],[1,6,4,8]]$ is proportional to the cross-product of the two vectors $\mathbf{c}_{128}$ and $\mathbf{c}_{318}=[[3,1,6,8],[3,1,4,8],[3,1,5,8]]$ whose coordinates appear in $T_{1,2,3,5}$ and $T_{1,2,4,5}$. More precisely, we have the following relation

$$
[1,2,3,8] \mathbf{c}_{18}=\mathbf{c}_{318} \times \mathbf{c}_{128}
$$

If we now change 8 in 7 , we immediately obtain that the vector $\mathbf{c}_{17}=[[1,4,5,7]$ $[1,5,6,7],[1,6,4,7]]$ is proportional to the cross-product of the two vectors $\mathbf{c}_{127}$ 
and $\mathbf{c}_{317}=[[3,1,6,7],[3,1,4,7],[3,1,5,7]]$ whose coordinates also appear in $T_{1,2,3,5}$ and $T_{1,2,4,5}$. More precisely, we have the following relation

$$
[1,2,3,7] \mathbf{c}_{17}=\mathbf{c}_{317} \times \mathbf{c}_{127}
$$

Note in passing that (20) and (21) can be obtrained from (18) and (19) by changing 1 into 2, 3 into 1 , and 2 into 3 . Since we have seen in section 4.3 how to recover the first two coordinates of $e_{3,1}$ from $T_{1,2,3,5}$ this shows, as advertised, that the coefficients of $T_{1,3,4,5}$ in group II can be very simply recovered from the coefficients of $T_{1,2,3,5}$ and $T_{1,2,4,5}$, in group $\mathrm{I}$.

To be complete, we summarize in table 3 the relations of the remaining coefficients of the trilinearities in group II to those of the trilinearities in the group I and in table 4 the relations of the coefficients of the trilinearities in group III to those of the trilinearities in the group I. For this last table, the notations are very similar to those used in table 3 . For example, the vector $\mathbf{d}_{25}$ has coordinates $[[2,5,7,8],[2,5,8,9],[2,5,9,6]]$, and the vector $\mathbf{d}_{125}$ has coordinates $[[1,2,5,9],[1,2,5,7],[1,2,5,8]]$. This completes the determination of

$$
\begin{aligned}
& {[1,2,3,7] \mathbf{c}_{17}=\mathbf{c}_{317} \times \mathbf{c}_{127}} \\
& {[1,2,3,8] \mathbf{c}_{18}=\mathbf{c}_{318} \times \mathbf{c}_{128}} \\
& {[1,2,3,9] \mathbf{c}_{19}=\mathbf{c}_{319} \times \mathbf{c}_{129}} \\
& {[1,2,3,7] \mathbf{c}_{27}=\mathbf{c}_{127} \times \mathbf{c}_{237}} \\
& {[1,2,3,8] \mathbf{c}_{28}=\mathbf{c}_{128} \times \mathbf{c}_{238}} \\
& {[1,2,3,9] \mathbf{c}_{29}=\mathbf{c}_{129} \times \mathbf{c}_{239}} \\
& {[1,2,3,7] \mathbf{c}_{37}=\mathbf{c}_{237} \times \mathbf{c}_{317}} \\
& {[1,2,3,8] \mathbf{c}_{38}=\mathbf{c}_{238} \times \mathbf{c}_{318}} \\
& {[1,2,3,9] \mathbf{c}_{39}=\mathbf{c}_{239} \times \mathbf{c}_{319}}
\end{aligned}
$$

Table 3: Relations of the coefficients of the trilinearities in group II with those in group I

the coefficients of the trilinearities in the groups II and III as functions of the coefficients of the trilinearities in the group I. 


$$
\begin{aligned}
& {[1,2,3,4] \mathbf{d}_{14}=\mathbf{d}_{124} \times \mathbf{d}_{314}} \\
& {[1,2,3,5] \mathbf{d}_{15}=\mathbf{d}_{125} \times \mathbf{d}_{315}} \\
& {[1,2,3,6] \mathbf{d}_{16}=\mathbf{d}_{126} \times \mathbf{d}_{316}} \\
& {[1,2,3,4] \mathbf{d}_{24}=\mathbf{d}_{234} \times \mathbf{d}_{124}} \\
& {[1,2,3,5] \mathbf{d}_{25}=\mathbf{d}_{235} \times \mathbf{d}_{125}} \\
& {[1,2,3,6] \mathbf{d}_{26}=\mathbf{d}_{236} \times \mathbf{d}_{126}} \\
& {[1,2,3,4] \mathbf{d}_{34}=\mathbf{d}_{314} \times \mathbf{d}_{234}} \\
& {[1,2,3,5] \mathbf{d}_{35}=\mathbf{d}_{315} \times \mathbf{d}_{235}} \\
& {[1,2,3,6] \mathbf{d}_{36}=\mathbf{d}_{316} \times \mathbf{d}_{236}}
\end{aligned}
$$

Table 4: Relations of the coefficients of the trilinearities in group III with those in group I

\subsection{Relation to previous work}

It is now a good time to relate our approach to previous ones, namely that of Hartley [12] and Shashua []. Since in reference [12] Richard Hartley relates his work to that of Shashua, we will relate ours to his and the reader can proceed by transitivity. Hartley introduces an entity depending upon three indexes which he denotes by $T_{i j k}, i, j, k=1,2,3$. To be consistent with his notations, let us denote the coordinates of a point $m_{1}$ in the first retina by $u_{k}, k=1,2,3$, those of a point $m_{2}$ in the second camera by $u_{k}^{\prime}, k=1,2,3$, and those of a point $m_{3}$ in the second camera by $u_{k}^{\prime \prime}, k=1,2,3$. Hartley then shows that if three points $m_{1}, m_{2}, m_{3}$ are the images of the same 3 -D point, then the four following quantities, obtained by varying the indexes $i$ and $l$ between 1 and 2 , are equal to 0 :

$$
T H S_{i, l} \equiv \sum_{k=1}^{3} u_{k}\left(u_{i}^{\prime} u_{l}^{\prime \prime} T_{k 33}-u_{3}^{\prime} u_{l}^{\prime \prime} T_{k i 3}-u_{i}^{\prime} u_{3}^{\prime \prime} T_{k 3 l}+u_{3}^{\prime} u_{3}^{\prime \prime} T_{k i l}\right)
$$

Hartley further assumes that $u_{3}=u_{3}^{\prime}=u_{3}^{\prime \prime}=1$.

In defining the matrix appearing in the left hand-side of equation (6), we have arbitrarily selected the first two equations appearing in (2) since the

$\operatorname{RR} \mathrm{n}^{\circ} 2665$ 
third one is linearly dependent of the first two and since it does not change the results. For the sake of consistency with Hartley's work, let us suppose that we redefine equation (6) in the following equivalent manner:

$$
\left[\begin{array}{c}
z_{1} \mathbf{1}-x_{1} \mathbf{3} \\
y_{1} \mathbf{3}-z_{1} \mathbf{2} \\
z_{2} \mathbf{4}-x_{2} \mathbf{6} \\
y_{2} \mathbf{6}-z_{2} \mathbf{5} \\
z_{3} 7-x_{3} \mathbf{9} \\
y_{3} \mathbf{9}-z_{3} 8
\end{array}\right] \mathbf{M}=\mathbf{0}
$$

and then use this equation to define the trilinearities. Looking at the table 1 , it should be clear that the four relations of Hartley (equation (8) in [12]) are exactly the trilinearities in the group I and that the correspondence between the $T_{i j k}$ 's and the brackets is extremely simple: $T_{i j k}$ is the bracket obtained by choosing the $i$ th element of the first column, the $k$ th element of the second, and the $l$ th element of the third column of the following "matrix":

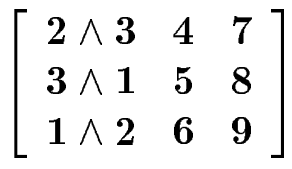

For example, $T_{111}=[2,3,4,7]$, and we have the formal correspondence:

$$
T H S_{1,1}=T_{1,2,3,5} \quad T H S_{1,2}=-T_{1,2,3,6} \quad T H S_{2,1}=-T_{1,2,4,5} \quad T H S_{2,2}=T_{1,2,4,6}
$$

This clearly shows the relationship between our work and Hartley's and, by transitivity, between our work and Shashua's. In particular, we think that the geometric interpretations of the trilinearities which are simple in our formalism shed a new light on the problem, making it much easier to grasp; this is a theoretical advantage. This theoretical advantage can perhaps be turned into a practical advantage as well since, as shown in sections 4.3 and 4.4, the geometric interpretation gives a natural minimal parametrization of the trilinearities.

In particular, we have seen what are the eight algebraic constraints on the 27 parameters upon which the four trilinearities in each group depend and what they mean geometrically. 


\section{Degenerate cases}

If the trilinearities were only useful to recover the epipolar geometry, then one may wonder why bother. We show now that they are in fact more powerful than the fundamental matrixes in the sense that they remain algebraically independent when the fundamental matrixes are not. To put it in another way, they can be used to predict the location of a third point in the third image given a pair of correspondences between the first two images even when the fundamental matrixes cannot be used to do so. This is already known thanks to Shashua's work []. In the following sections, we simply show that our formalism naturally yields the same results.

\subsection{Optical centers not aligned}

In this configuration, it is known that if the point $M$ is in the trifocal plane (the plane defined by the three optical centers), its image in one of the cameras, for example $m_{3}$, cannot be predicted from its images $m_{1}$ and $m_{2}$ in the other two cameras [31]. The traces of this plane in the three retinal planes are the three trifocal lines $\left\langle e_{i, i+1}, e_{i, i+2}\right\rangle, i=1,2,3$. This is because the epipolar lines of $m_{1}$ and $m_{2}$ in the third image are identical and therefore $m_{3}$ cannot be obtained as their intersection: $m_{3}$ is undefined on the common epipolar line. But the trilinear relations can be used to compute the position of $m_{3}$ on this line. To show this, let us assume that $\mathbf{m}_{1}=a_{1} \mathbf{e}_{1,2}+b_{1} \mathbf{e}_{1,3}, \mathbf{m}_{2}=a_{2} \mathbf{e}_{2,1}+b_{2} \mathbf{e}_{2,3}$, $\mathbf{m}_{3}=a_{3} \mathbf{e}_{3,1}+b_{3} \mathbf{e}_{3,2}$ are on the trifocal lines. In this case, the bilinear relations are satisfied whatever the values of the $a_{i}, b_{i}$ are. Substituting these relations in $T_{1,3,5,6}$, taking the normal form (as standard monomials in determinants),

$\operatorname{RR} \mathrm{n}^{\circ} 2665$ 
we obtain:

$$
\begin{aligned}
& a_{1} b_{2} a_{3}[1,2,3,5][1,2,3,6][4,5,7,8][4,7,8,9] \\
& \quad+b_{1} a_{2} b_{3}[1,2,3,5][1,2,3,6][4,5,7,8][4,7,8,9] \\
& \quad-a_{1} b_{2} a_{3}[1,2,3,5]^{2}[4,6,7,8][4,7,8,9] \\
& \quad-b_{1} a_{2} b_{3}[1,2,3,5]^{2}[4,6,7,8][4,7,8,9] \\
& \quad-a_{1} b_{2} a_{3}[1,2,3,4][1,2,3,6][4,5,7,8][5,7,8,9] \\
& \quad-b_{1} a_{2} b_{3}[1,2,3,4][1,2,3,6][4,5,7,8][5,7,8,9] \\
& \quad+2 a_{1} b_{2} a_{3}[1,2,3,4][1,2,3,5][4,6,7,8][5,7,8,9] \\
& \quad+2 b_{1} a_{2} b_{3}[1,2,3,4][1,2,3,5][4,6,7,8][5,7,8,9] \\
& \quad-a_{1} b_{2} a_{3}[1,2,3,4][1,2,3,5][4,5,7,8][6,7,8,9] \\
& \quad-b_{1} a_{2} b_{3}[1,2,3,4][1,2,3,5][4,5,7,8][6,7,8,9] \\
& \quad-a_{1} b_{2} a_{3}[1,2,3,4]^{2}[5,6,7,8][5,7,8,9] \\
& \quad-b_{1} a_{2} b_{3}[1,2,3,4]^{2}[5,6,7,8][5,7,8,9]=0
\end{aligned}
$$

This fairly ugly polynomial can be rewritten more simply as:

$$
a_{3} P\left(a_{1}, b_{1}, a_{2}, b_{2}\right)+b_{3} Q\left(a_{1}, b_{1}, a_{2}, b_{2}\right)=0
$$

where $P$ and $Q$ are two non zero homogeneous polynomials of degree 2 in $a_{1}, b_{1}, a_{2}, b_{2}$. They are nonzero because the bracket monomials are nonzero since they are in normal form.

Consequently, knowing the position of any two points (for example $m_{1}$ and $m_{2}$ ) on the epipolar lines, the third (for example $m_{3}$ ) is completely determined by the previous equation.

\subsection{Optical centers aligned}

In this configuration, the epipoles $e_{i, i-1}$ and $e_{i, i+1}$ are identical and the epipolar lines of two corresponding points $m_{1}$ and $m_{2}$ in the third image are always identical. The corresponding point $m_{3}$ is thus undefined on this epipolar line. But, just as in the previous case, the trilinear relations can be used to compute the position of $m_{3}$ on this line. As the centers are on a same line $L$, we can assume that this line is the line $\mathbf{1} \wedge \mathbf{2}$ and that the planes 4 and 7 (resp. (6 and $\mathbf{9}$ ) are identical to the plane $\mathbf{1}$ (resp. 2). 
Let us denote by $L_{1}$ the expression $x_{1} \mathbf{2} \wedge \mathbf{3}+y_{1} \mathbf{3} \wedge \mathbf{1}+z_{1} \mathbf{1} \wedge \mathbf{2}$. It is easy to show that

$$
\begin{aligned}
& T_{1,2,3,5}=x_{2} x_{3} L_{1} \wedge \mathbf{5} \wedge \mathbf{8}-x_{1}\left(x_{2} y_{3}[1,2,3,5]-y_{2} x_{3}[1,2,3,8]\right) \\
& T_{1,2,4,6}=z_{2} z_{3} L_{1} \wedge \mathbf{5} \wedge \mathbf{8}-z_{1}\left(x_{2} y_{3}[1,2,3,5]-y_{2} x_{3}[1,2,3,8]\right)
\end{aligned}
$$

Looking at equation (4), it is seen that $\mathbf{F}_{1,2}$ is proportional to

$$
\left[\begin{array}{ccc}
0 & -1 & 0 \\
0 & 0 & 0 \\
1 & 0 & 0
\end{array}\right]
$$

Thus, if we take $x_{2}=x_{1}$ and $z_{2}=y_{1}, m_{1}$ and $m_{2}$ correspond to each other. We then have:

$$
\begin{aligned}
& T_{1,2,3,5}=x_{1}\left(x_{3}\left(L_{1} \wedge \mathbf{5} \wedge \mathbf{8}-y_{2}[1238]\right)-y_{3} x_{1}[1235]\right) \\
& T_{1,2,4,6}=z_{3} y_{1} L_{1} \wedge \mathbf{5} \wedge \mathbf{8}-z_{1}\left(x_{1} y_{3}[1235]-y_{2} x_{3}[1238]\right)
\end{aligned}
$$

Except if $x_{1}=0$, the equation $T_{1,2,3,5}=0$ can be used to compute the ratio of $x_{3}$ and $y_{3}$ and, except if $y_{1}=0$, the equation $T_{1,2,4,6}=0$ can be used to recover the ratio of $z_{3}$ and $y_{3}$. This determines the third point from the first two.

\section{Quadrilinear constraints}

Let us now add a fourth image. Equation (6) can be written

$$
\left[\begin{array}{c}
x_{1} \mathbf{2}-y_{1} \mathbf{1} \\
y_{1} \mathbf{3}-z_{1} \mathbf{2} \\
x_{2} \mathbf{5}-y_{2} \mathbf{2} \\
y_{2} \mathbf{6}-z_{2} \mathbf{5} \\
x_{3} 8-y_{3} 7 \\
y_{3} \mathbf{9}-z_{3} 8 \\
x_{4} \mathbf{1 1}-y_{4} \mathbf{1 0} \\
y_{4} \mathbf{1 2}-z_{4} \mathbf{1 1}
\end{array}\right] \mathbf{M}=\mathbf{0}
$$

where we have again chosen arbitrarily the first two equations in (2) for each camera. The results to come are independent of this choice. 
The $8 \times 4$ matrix which appears in this equation is a linear function of the four points $m_{1}, m_{2}, m_{3}, m_{4}$. The question is, what is the necessary and sufficient condition that the four points $m_{i}$ must satisfy so that they can be considered as the images of the same point $M$. The answer comes again from elementary linear algebra and is that the $8 \times 4$ matrix must be of rank less than or equal to three. This is equivalent to writing that all its $4 \times 4$ subdeterminants are zero. There are three types of such determinants:

1. Those which contain two rows arising from one camera and two rows from another.

2. Those which contain two rows arising from one camera, one row from a second camera and one row arising from a third camera.

3. Those which contain one row arising from each camera.

There are six determinants of the first type and, as seen in the previous sections, they correspond to the six fundamental matrixes of the six pairs of cameras. There are 32 determinants of the second type and 16 determinants of the fourth type. Computing for example the one built from the first, third, fifth, and seventh rows, which we note $Q_{1,3,5,7}$, in agreement with our notation for trilinearities, we find the following expression

$$
\begin{aligned}
x_{1} & x_{2} x_{3} x_{4}[2,5,8,11]-x_{1} x_{2} x_{3} y_{4}[2,5,8,10] \\
& -x_{1} x_{2} y_{3} x_{4}[2,5,7,11]+x_{1} x_{2} y_{3} y_{4}[2,5,7,10] \\
- & x_{1} y_{2} x_{3} x_{4}[2,4,8,11]+x_{1} y_{2} x_{3} y_{4}[2,4,8,10] \\
+ & x_{1} y_{2} y_{3} x_{4}[2,4,7,11]-x_{1} y_{2} y_{3} y_{4}[2,4,7,10] \\
& -y_{1} x_{2} x_{3} x_{4}[1,5,8,11]+y_{1} x_{2} x_{3} y_{4}[1,5,8,10] \\
+ & y_{1} x_{2} y_{3} x_{4}[1,5,7,11]-y_{1} x_{2} y_{3} y_{4}[1,5,7,10] \\
+ & y_{1} y_{2} x_{3} x_{4}[1,4,8,11]-y_{1} y_{2} x_{3} y_{4}[1,4,8,10] \\
& -y_{1} y_{2} y_{3} x_{4}[1,4,7,11]+y_{1} y_{2} y_{3} y_{4}[1,4,7,10]
\end{aligned}
$$

This is seen to be a polynomial of total degree four in the coordinates of the points $m_{i}$ and linear in the coordinates of each of them. It is a quadrilinear relation which has been reported by Triggs [29]. The geometrical interpretation of this computation is as follows: $x_{1} \mathbf{2}-y_{1} \mathbf{1}, \ldots$ represent lines in the images, 
which are the intersections of the retinal planes with planes through the optical centers of the cameras in $\mathbb{P}^{3}$. The vanishing of the quadrilinear polynomial is just the condition that these four planes have a common point in $\mathbb{P}^{3}$.

Assuming that all the bilinear and trilinear relations are satisfied, it is possible to show that the quadrilinear relations are satisfied because they can be obtained as linear combinations of the previous ones. Indeed, this is also a consequence of the Plücker relations or Cramer's rule. Let consider for instance the 4 dimensional rows $\mathbf{C}_{1}, \mathbf{C}_{2}, \mathbf{C}_{3}, \mathbf{C}_{5}, \mathbf{C}_{7}$ of the matrix (25). They are connected by the Cramer relation

$$
\begin{aligned}
& {\left[\mathbf{C}_{2}, \mathbf{C}_{3}, \mathbf{C}_{5}, \mathbf{C}_{7}\right] \mathbf{C}_{1}-\left[\mathbf{C}_{1}, \mathbf{C}_{3}, \mathbf{C}_{5}, \mathbf{C}_{7}\right] \mathbf{C}_{2}+\left[\mathbf{C}_{1}, \mathbf{C}_{2}, \mathbf{C}_{5}, \mathbf{C}_{7}\right] \mathbf{C}_{3}} \\
& -\left[\mathbf{C}_{1}, \mathbf{C}_{2}, \mathbf{C}_{3}, \mathbf{C}_{7}\right] \mathbf{C}_{5}+\left[\mathbf{C}_{1}, \mathbf{C}_{2}, \mathbf{C}_{3}, \mathbf{C}_{5}\right] \mathbf{C}_{7}=0
\end{aligned}
$$

Multiplying for instance by $\mathbf{C}_{1} \wedge \mathbf{5} \wedge \mathbf{6}$ we obtain a relation of the form

$$
\begin{aligned}
& {\left[\mathbf{C}_{1}, \mathbf{C}_{3}, \mathbf{C}_{5}, \mathbf{C}_{7}\right]\left[\mathbf{C}_{1}, \mathbf{C}_{2}, \mathbf{5}, \mathbf{6}\right]-\left[\mathbf{C}_{1}, \mathbf{C}_{2}, \mathbf{C}_{3}, \mathbf{C}_{7}\right]\left[\mathbf{C}_{1}, \mathbf{C}_{5}, \mathbf{5}, \mathbf{6}\right]} \\
& \quad+\left[\mathbf{C}_{1}, \mathbf{C}_{2}, \mathbf{C}_{3}, \mathbf{C}_{5}\right]\left[\mathbf{C}_{1}, \mathbf{C}_{7}, \mathbf{5}, \mathbf{6}\right]=0
\end{aligned}
$$

Remark that the first term $\left[\mathbf{C}_{1}, \mathbf{C}_{3}, \mathbf{C}_{5}, \mathbf{C}_{7}\right]$ is the previous quadrilinearity and the other are trilinearities. More precisely, the previous equation can be rewritten as:

$$
y_{1}\left(Q_{1,3,5,7}\left(\mathbf{F}_{1,2} \mathbf{m}_{1}\right)_{1}-T_{1,2,3,7} \operatorname{coeff}\left(T_{1,3,4,5}, y_{2}\right)-T_{1,2,3,5} \operatorname{coeff}\left(T_{1,3,4,7}, y_{2}\right)\right)=0
$$

This is a "generic" linear relation between the quadrilinearities and the trilinearities. Hence the quadrilinear relations do not add more information.

It turns out that the four cameras case is the generic one since if we consider more than four cameras the same reasoning as before will show that there are no other relations than those that we have already described, bilinear, trilinear, and quadrilinear and that the quadrilinear are dependent of the first two classes.

\section{The case of lines}

The case of lines is also important since they are quite often present in 3-D scenes and therefore in images. Hartley [12] has started analysing the case of 
line correspondences. We provide here a complete treatment and relate it to the case of point correspondences. In order to do this, we first generalise the perspective projection matrix, defined in the case of points, to lines. Given two $3-\mathrm{D}$ points $M_{1}$ and $M_{2}$, the line $L \equiv\left\langle M_{1}, M_{2}\right\rangle$ can be represented by its Plücker coordinates, noted $\mathbf{L}=\mathbf{M}_{1} \wedge \mathbf{M}_{2}=\left[L^{12}, L^{13}, L^{14}, L^{23}, L^{24}, L^{34}\right]^{T}$, a $6 \times 1$ vector whose coordinates are defined up to a scale factor and satisfy the following quadratic equation

$$
[\mathbf{L}, \mathbf{L}] \equiv L^{12} L^{34}-L^{13} L^{24}+L^{14} L^{23}=0
$$

Now, given the line $L \equiv\left\langle M_{1}, M_{2}\right\rangle$, its image $l$ through a camera defined by the perspective projection matrix $\mathbf{P}$ is represented by the $3 \times 1$ vector:

$$
\begin{aligned}
& \mathbf{1}=\mathbf{P M}_{1} \times \mathbf{P} \mathbf{M}_{2}= \\
& \left(\left(\mathbf{2} \cdot \mathbf{M}_{1}\right)\left(\mathbf{3} \cdot \mathbf{M}_{2}\right)-\left(\mathbf{3} \cdot \mathbf{M}_{2}\right)\left(\mathbf{2} \cdot \mathbf{M}_{1}\right),\left(\mathbf{3} \cdot \mathbf{M}_{1}\right)\left(\mathbf{1} \cdot \mathbf{M}_{2}\right)-\left(\mathbf{1} \cdot \mathbf{M}_{2}\right)\left(\mathbf{3} \cdot \mathbf{M}_{1}\right),\right. \\
& \left.\left(\mathbf{1} \cdot \mathbf{M}_{1}\right)\left(\mathbf{2} \cdot \mathbf{M}_{2}\right)-\left(\mathbf{2} \cdot \mathbf{M}_{2}\right)\left(\mathbf{1} \cdot \mathbf{M}_{1}\right)\right)^{T}
\end{aligned}
$$

Each coordinate of this vector is antisymmetric in $\mathbf{M}_{1}, \mathbf{M}_{2}$. Moreover, the first coordinate is antisymmetric in $\mathbf{2}, \mathbf{3}$, the second in $\mathbf{3}, \mathbf{1}$ and the third in $\mathbf{1}, \mathbf{2}$. It is easy to verify that the image line $d$, image of the line $L$ is represented by the vector $\mathbf{d}$ of coordinates

$$
[(\mathbf{2} \wedge \mathbf{3}) \cdot \mathbf{L},(\mathbf{3} \wedge \mathbf{1}) \cdot \mathbf{L},(\mathbf{1} \wedge \mathbf{2}) \cdot \mathbf{L}]^{T}
$$

We can rewrite this linearly in terms of Plücker coordinates as

$$
\mathbf{d}=\tilde{\mathbf{P}} \mathbf{L}
$$

where $\tilde{\mathbf{P}}$ is the following $3 \times 6$ matrix:

$$
\left[\begin{array}{l}
2 \wedge \mathbf{3} \\
3 \wedge \mathbf{1} \\
\mathbf{1} \wedge \mathbf{2}
\end{array}\right]
$$

The matrix $\tilde{\mathbf{P}}$ plays for $3-\mathrm{D}$ lines the same role that the matrix $\mathbf{P}$ plays for 3-D points.

Equation (29) is thus equivalent to

$$
l_{1}: l_{2}: l_{3}=(\mathbf{2} \wedge \mathbf{3}) \cdot \mathbf{L}:(\mathbf{3} \wedge \mathbf{1}) \cdot \mathbf{L}:(\mathbf{1} \wedge \mathbf{2}) \cdot \mathbf{L}
$$


We know that this yields two independent equations, for example

$$
\left[\begin{array}{l}
l_{1} \mathbf{3} \wedge \mathbf{1}-l_{2} \mathbf{2} \wedge \mathbf{3} \\
l_{2} \mathbf{1} \wedge \mathbf{2}-l_{3} \mathbf{3} \wedge \mathbf{1}
\end{array}\right] \mathbf{L}=\mathbf{0}
$$

Each row of the matrix that appears in the left hand-side of this equation represents a line going through the optical center of the camera and through a point of the image line $d$. It can be readily verified that each row vector satisfies the equation (28) and therefore represents a line. In fact, we have for instance $l_{2} \mathbf{1} \wedge \mathbf{2}-l_{3} \mathbf{3} \wedge \mathbf{1}=\mathbf{1} \wedge\left(l_{2} \mathbf{2}+l_{3} \mathbf{3}\right)$.

Let us now consider a second camera defined by its perspective matrix $\mathbf{P}_{2}$ and the corresponding matrix $\tilde{\mathbf{P}}_{2}$. We can now ask the same question as for points: given a line $d$ in the first image and a line $d^{\prime}$ in the second one, what are the necessary and sufficient conditions that these lines must satisfy in order to be the images of the same 3-D line $L$ ? These conditions would be the equivalent of the epipolar constraint in the case of points. The previous analysis shows that we have four equations like the ones above:

$$
\left[\begin{array}{l}
l_{1} \mathbf{3} \wedge \mathbf{1}-l_{2} \mathbf{2} \wedge \mathbf{3} \\
l_{2} \mathbf{1} \wedge \mathbf{2}-l_{3} \mathbf{3} \wedge \mathbf{1} \\
l_{1}^{\prime} \mathbf{6} \wedge \mathbf{4}-l_{2}^{\prime} \mathbf{5} \wedge \mathbf{6} \\
l_{2}^{\prime} 4 \wedge \mathbf{5}-l_{3}^{\prime} \mathbf{6} \wedge \mathbf{4}
\end{array}\right] \mathbf{L}=\mathbf{0}
$$

The matrix which appears on the left hand-side is $4 \times 6$, hence its kernel is of dimension larger than or equal to 2 . Therefore there is no condition and two image lines can always be considered as the images of a 3-D line, a well-known fact in computer vision.

Things become interesting when a third camera comes in because we now have six independent equations:

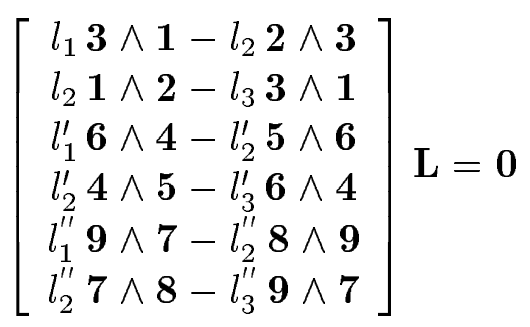

$\operatorname{RR} \mathrm{n}^{\circ} 2665$ 
The necessary and sufficient conditions for the three lines $d, d^{\prime}, d^{\prime \prime}$ to be the images of the same 3 -D line are that a) the $6 \times 6$ matrix which appears on the left hand-side of the previous equation is of rank less than or equal to 5 , and b) that there exists in its kernel a vector $\mathbf{L}$ which represents a line, i.e. which satisfies equation (28). The first condition is equivalent to writing that its determinant is zero. We note that this determinant is a polynomial of total degree 6 in the coordinates of the three lines $d, d^{\prime}, d^{\prime \prime}$ and that it is divisible by the monomial $l_{2} l_{2}^{\prime} l_{2}^{\prime \prime}$. The remaining polynomial is of degree 3 in the coordinates of the three lines, a trilinear relation whose coefficients can be expressed in terms of the row vectors of the perspective projection matrixes $\mathbf{P}_{1}, \mathbf{P}_{2}, \mathbf{P}_{3}$.

The second condition is equivalent to the condition that the six $5 \times 5$ minors of the $6 \times 5$ matrix obtained by erasing any of the rows of the previous $6 \times 6$ matrix satisfy equation (28). This condition is a bit tricky to write and this is the reason why we have preferred a slightly different approach. Let $L_{i}, i=1, \cdots, 6$ be the six lines represented by the rows of the previous $6 \times 6$ matrix. $L_{3}$ and $L_{4}$ go through the second optical center, and they define a plane going through the optical center of the second image which is, in terms of the Grassmann-Cayley formalism, their join $L_{3} \vee L_{4}$. This plane is represented by $l_{1}^{\prime} 4+l_{2}^{\prime} \mathbf{5}+l_{3}^{\prime} \mathbf{6}$. Similarly, $L_{5} \vee L_{6}$ defines a plane represented by $l_{1}^{\prime \prime} 6+l_{2}^{\prime \prime} \mathbf{7}+l_{3}^{\prime \prime} 8$ going through the optical center of the third camera and through the image line $d^{\prime \prime}$. These two planes meet at a line $L$ which is their meet $\left(L_{3} \vee L_{4}\right) \wedge\left(L_{5} \vee L_{6}\right)$. The conditions we seek are that the two lines $L_{1}$ and $L_{2}$ meet $L$. Hence, we obtain the two scalar equations:

$$
\begin{aligned}
& L_{1} \wedge\left(\left(L_{3} \vee L_{4}\right) \wedge\left(L_{5} \vee L_{6}\right)\right)=0 \\
& L_{2} \wedge\left(\left(L_{3} \vee L_{4}\right) \wedge\left(L_{5} \vee L_{6}\right)\right)=0
\end{aligned}
$$

Each one of these conditions is seen to be a polynomial of total degree 5 in the coordinates of the image lines $d, d^{\prime}, d^{\prime \prime}$. Since both polynomials are divisible by the monomial $l_{2}^{\prime} l_{2}^{\prime \prime}$, the two conditions are actually of total degree 3 , the monomial being a consequence of the choice of the particular equations in building the $6 \times 6$ matrix.

According to the previous analysis, the two conditions can be rewritten as:

$$
\begin{aligned}
& \left(l_{1} \mathbf{3} \wedge \mathbf{1}-l_{2} \mathbf{2} \wedge \mathbf{3}\right) \wedge\left(l_{1}^{\prime} 4+l_{2}^{\prime} 5+l_{3}^{\prime} 6\right) \wedge\left(l_{1}^{\prime \prime} 6+l_{2}^{\prime \prime} 7+l_{3}^{\prime \prime} 8\right)=0 \\
& \left(l_{2} \mathbf{1} \wedge \mathbf{2}-l_{3} \mathbf{3} \wedge \mathbf{1}\right) \wedge\left(l_{1}^{\prime} 4+l_{2}^{\prime} 5+l_{3}^{\prime} 6\right) \wedge\left(l_{1}^{\prime \prime} 6+l_{2}^{\prime \prime} 7+l_{3}^{\prime \prime} 8\right)=0
\end{aligned}
$$


Using the notations of section 4.6, they can be rewritten as:

$$
\begin{aligned}
& l_{1} \sum_{i, j=1}^{3} T_{2 i j} l_{i}^{\prime} l_{j}^{\prime \prime}-l_{2} \sum_{i, j=1}^{3} T_{1 i j} l_{i}^{\prime} l_{j}^{\prime \prime}=0 \\
& l_{2} \sum_{i, j=1}^{3} T_{3 i j} l_{i}^{\prime} l_{j}^{\prime \prime}-l_{3} \sum_{i, j=1}^{3} T_{2 i j} l_{i}^{\prime} l_{j}^{\prime \prime}=0
\end{aligned}
$$

From which we see that we have:

$$
l_{1}: l_{2}: l_{3}=\sum_{i, j=1}^{3} T_{1 i j} l_{i}^{\prime} l_{j}^{\prime \prime}: \sum_{i, j=1}^{3} T_{2 i j} l_{i}^{\prime} l_{j}^{\prime \prime}: \sum_{i, j=1}^{3} T_{3 i j} l_{i}^{\prime} l_{j}^{\prime \prime}
$$

which is precisely the expression found by Hartley [12] and relates the line trilinearities to the point trilinearities in the group I through the relations (24).

\section{Conclusion}

We have shown that the geometry of the correspondences between the images of a single 3 -D point in $N$ cameras can be described by three types of relations between the coordinates of the image points. These relations fall into three classes of which only the first two are sufficient since all elements in the third one are algebraically dependent of elements in the first two. The coefficients of these relations have been shown to be $4 \times 4$ determinants of the row vectors of the perspective projection matrixes of the cameras.

We have shown that the trilinear relations are useful in some degenerate cases of practical importance where the bilinear relations cannot be used for prediction.

We have started the analysis of the algebraic constraints between the trilinear and the bilinear constraints and shown that the trilinear constraints imply the bilinear ones. Finally, we have shown how to extend this analysis to lines

correspondences. All this has been achieved by simple symbolic computations in projective geometry using the Grassmann-Cayley algebra formalism.

\section{Acknowledgements}

The authors gratefully acknowledge the contributions of Didier Bondyfalat and Stéphane Laveau.

$\operatorname{RR} \mathrm{n}^{\circ} 2665$ 


\section{A Cramer's relation}

We derive Cramer's relation for five vectors of a vector space of dimension 4. It should be clear that the method is extendable to any dimension. Let $\mathbf{C}_{1}, \mathbf{C}_{2}, \mathbf{C}_{3}, \mathbf{C}_{4}$ and $\mathbf{C}_{5}$ be five vectors of a vector space of dimension 4 . Since they are linearly dependent, there exist five scalars $\lambda_{i}, i=1, \cdots, 5$ not all equal to zero such that:

$$
\sum_{i=1}^{5} \lambda_{i} \mathbf{C}_{i}=\mathbf{0}
$$

We compute the ratios $\lambda_{i} / \lambda_{5}, i=1, \cdots, 4$ by taking the exterior product of this equation successively by $\mathbf{C}_{2} \wedge \mathbf{C}_{3} \wedge \mathbf{C}_{4}, \mathbf{C}_{1} \wedge \mathbf{C}_{3} \wedge \mathbf{C}_{4}, \mathbf{C}_{1} \wedge \mathbf{C}_{2} \wedge \mathbf{C}_{4}$, and $\mathbf{C}_{1} \wedge \mathbf{C}_{2} \wedge \mathbf{C}_{3}$. We finally obtain the desired formula:

\section{$\left[\mathbf{C}_{2} \wedge \mathbf{C}_{3} \wedge \mathbf{C}_{4} \wedge \mathbf{C}_{5}\right] \mathbf{C}_{1}-\left[\mathbf{C}_{1} \wedge \mathbf{C}_{3} \wedge \mathbf{C}_{4} \wedge \mathbf{C}_{5}\right] \mathbf{C}_{2}+\left[\mathbf{C}_{1} \wedge \mathbf{C}_{2} \wedge \mathbf{C}_{4} \wedge \mathbf{C}_{5}\right] \mathbf{C}_{3}-$$$
\left[\mathbf{C}_{1} \wedge \mathbf{C}_{2} \wedge \mathbf{C}_{3} \wedge \mathbf{C}_{5}\right] \mathbf{C}_{4}+\left[\mathbf{C}_{1} \wedge \mathbf{C}_{2} \wedge \mathbf{C}_{3} \wedge \mathbf{C}_{4}\right] \mathbf{C}_{5} \neq 310
$$ \\ B Some basics about the Grassmann-Cayley algebra}

The ambient space where we want to work is a projective space $\mathbb{P}$ of dimension $n$ (over the field $\mathbf{k}$ ). After taking a referential, we can consider it as the projective space associated to a $\mathbf{k}$-vector space $\mathbb{E}$ of dimension $n+1$. Let $\underline{e}=\left(e_{0}, \ldots, e_{n}\right)$ be a basis of $\mathbb{E}$. If $\left(u_{0}, \ldots, u_{n}\right)$ is a non-zero vector of $\mathbb{E}$, we will denote by $u=\left(u_{0}: \cdots: u_{n}\right)$ the element of the projective space $\mathbb{P}=\mathbb{P}(\mathbb{E})$ associated to it (that is the set of $(n+1)$-tuples equal to $\left(u_{0}, \ldots, u_{n}\right)$ up to multiplication by a non-zero scalar). We will denote by $\mathbf{u}$ the multiple $\frac{1}{u_{0}}\left(u_{0}, \ldots, u_{n}\right)$ of $u$ (if $u_{0} B \neq 0$ ) such that the first coordinate is 1 .

We want to manipulate points, lines, planes, etc of this space. For this purpose let recall the construction of the exterior algebra $\wedge \mathbb{E}$ of $\mathbb{E}$ (see [5]). The exterior algebra $\wedge \mathbb{E}$ is the k-algebra space generated by the "words" on the alphabet $e_{0}, \ldots, e_{n}$ denoted by

$$
e_{i_{1}} \wedge \cdots \wedge e_{i_{k}} \text { with } 0 \leq i_{j} \leq n
$$

(where $\wedge$ is the multiplication operator or concatenation) modulo the following relations: 
1. $\wedge$ is bilinear: $e_{i} \wedge\left(\lambda e_{j}+\mu e_{k}\right)=\lambda e_{i} \wedge e_{j}+\mu e_{i} \wedge e_{k}$,

2. $\wedge$ is anti-commutative: $e_{i} \wedge e_{j}=-e_{j} \wedge e_{i}$.

This implies that

$$
\wedge \mathbb{E}=\mathbf{k} \oplus \mathbb{E} \oplus \wedge^{2} \mathbb{E} \oplus \cdots \oplus \wedge^{n+1} \mathbb{E}
$$

where $\wedge^{k} \mathbb{E}$ is the $\mathrm{k}$-vector space generated by the terms $e_{i_{1}} \wedge \cdots \wedge e_{i_{k}}$ (with $\left.i_{1}<\cdots<i_{k}\right)$. An element of $\wedge^{k} \mathbb{E}$ is said to be of degree $k$.

The dimension of $\wedge \mathbb{E}$ is $2^{n+1}$, a basis is $\left(e_{i_{1}} \wedge \cdots \wedge e_{i_{k}}\right)_{1<i_{1}<\cdots<i_{m} \leq n+1}$ and $\wedge \mathbb{E}$ contains $\mathbb{E}$ and $\mathbf{k}$. The coordinates of an element $L$ of $\wedge^{k+1} \mathbb{E}$ in the basis $e_{i_{0}} \wedge \cdots \wedge e_{i_{k}}$ are denoted by $\left(L\left[i_{0}, \ldots, i_{k}\right]\right)$.

For all vectors $u_{1}=\left(u_{1,1}, \ldots, u_{n+1,1}\right), \ldots, u_{m}=\left(u_{1, m}, \ldots, u_{n+1, m}\right)$ of $\mathbb{E}$, the coordinates of $u_{1} \wedge \cdots \wedge u_{m}$ in the basis $\left(e_{i_{1}} \wedge \cdots \wedge e_{i_{m}}\right)_{1 \leq i_{1}<\cdots<i_{m} \leq n+1}$ of $\wedge^{m} \mathbb{E}$ are the corresponding determinants $\left|u_{i_{k}, j}\right|_{1 \leq i, k \leq m}$. Writing " $u_{1} \wedge \cdots \wedge u_{m}=0$ " is equivalent to saying that $\left\{u_{1}, \ldots, u_{m}\right\}$ are linearly dependents.

We denote by $\left|u_{1}, \ldots, u_{n}\right|$ the determinant of the vectors $u_{1}, \ldots, u_{n}$ in the basis $\underline{e}$.

\section{The Grassmannian}

The space of representation of linear spaces, that we are going to use, is the projective space $\mathbb{P}(\wedge \mathbb{E})$ associated to this $\mathrm{k}$-vector space. For any element $L \in \wedge \mathbb{E}$, we define $\vec{L}$ as the set of vector $v \in \mathbb{E}$ such that $v \wedge L=0$. A point $v$ is in the space $\left\langle v_{0}, \ldots, v_{d}\right\rangle$ if and only if it satisfies

$$
v \wedge v_{0} \wedge \cdots \wedge v_{k}=0
$$

Consequently if $L=v_{1} \wedge \cdots \wedge v_{k}$, the vector space $\vec{L}$ is the space generated by $v_{1}, \ldots, v_{k}$. It is independent of the representation $v_{1} \wedge \cdots \wedge v_{k}$, that we have chosen for $L$. If we choose another basis $w_{1}, \ldots, w_{k}$ of $\vec{L}$, we have the relation $w_{0} \wedge \cdots \wedge w_{k}=\operatorname{det}_{\underline{v}}(\underline{w}) L$ where $\operatorname{det}_{\underline{v}}(\underline{w}) \neq 0$ is the determinant of the basis $\underline{w}$ in $\underline{v}$. This gives us a one-to-one correspondence between the subspaces of $\mathbb{E}$ of dimension $k$ and the element of $\wedge \mathbb{E}$ of the form $v_{1} \wedge \cdots \wedge v_{k}$.

In $\mathbb{E}_{4}$, the line generated by two points $\mathbf{a}=\left(1, a_{1}, a_{2}, a_{3}\right)$ and $\mathbf{b}=\left(1, b_{1}, b_{2}, b_{3}\right)$ is the element of $\wedge^{2} \mathbb{E}$ of coordinates the $2 \times 2$ minors of the matrix

$$
\left[\begin{array}{llll}
1 & a_{1} & a_{2} & a_{3} \\
1 & b_{1} & b_{2} & b_{3}
\end{array}\right]
$$

$\operatorname{RR} \mathrm{n}^{\circ} 2665$ 
This yields, up to a sign, the 3 coordinates of the vector $\mathbf{a} \overrightarrow{\mathbf{b}}$ and the 3 coordinates of $\overrightarrow{\mathbf{o a}} \times \mathbf{o} \overrightarrow{\mathbf{b}}$ where $\mathbf{o}=e_{0}$ is the origin of the frame and $\times$ is the vector product.

In this representation, points in $\mathbb{P}(\mathbb{E})$ are elements of degree 1 in $\mathbb{P}(\wedge \mathbb{E})$, lines are of degree 2, planes of degree 3 and so on.

Definition B.1 - The subset of $\mathbb{P}\left(\wedge^{k} \mathbb{E}\right)$ of elements of the form $v_{0} \wedge \cdots \wedge v_{k}$ is called the Grassmannian $\mathcal{G}_{k}(\mathbb{E})$ of $k$-spaces in $\mathbb{E}$.

The coordinates of an element of the form $v_{1} \wedge \cdots \wedge v_{k}$ are the determinants $k \times k$ of the matrix $\left[v_{0}, \ldots, v_{k}\right]$ and satisfy the well-known Plücker relations (see [6], [14]). We denote by $\mathcal{G}$ the union of all $\mathcal{G}_{k}(\mathbb{E})$ for $1 \leq k \leq n$.

The lines in a space $\mathbb{P}_{3}=\mathbb{P}\left(\mathbb{E}_{4}\right)$ (where $e_{0}, \ldots, e_{3}$ is a basis of $\mathbb{E}_{4}$ ) are represented by elements of $\mathbb{P}\left(\wedge^{2} \mathbb{E}\right)$. The coordinates of an element $L \in \mathcal{G}_{2}$ in the basis $e_{0} \wedge e_{1}, e_{0} \wedge e_{2}, e_{1} \wedge e_{2}, e_{0} \wedge e_{3}, e_{1} \wedge e_{3}, e_{2} \wedge e_{3}$ are denoted by $\left(L_{0,1}: L_{0,2}: L_{1,2}: L_{0,3}: L_{1,3}: L_{2,3}\right)$. A line through two points $a, b$ will be represented by $L=a \wedge b$. Its coordinates (which are the $2 \times 2$ minors of the matrix $[a, b])$ satisfy the relation

$$
L_{0,1} L_{2,3}-L_{0,2} L_{1,3}+L_{0,3} L_{1,2}=0 \text {. }
$$

Conversely an element of $\mathbb{P}\left(\wedge^{2} \mathbb{E}\right)$ which satisfies this relation is of the form $a \wedge b$. The elements satisfying this relation form the Grassmannian $\mathcal{G}_{2}$ of $\mathbb{P}_{3}$.

The quadratic form (32) defines a non-degenerate bilinear product (denoted by $[\mid])$ and we have the following properties:

- For every element $L$ of $\mathbb{P}\left(\wedge^{2} \mathbb{E}\right),[L \mid L]=0$ if and only if $L \in \mathcal{G}_{2}$.

- For every points $a, b, c, d \in \mathbb{P},[a \wedge b \mid c \wedge d]=\operatorname{det}(a, b, c, d)$.

- For every lines in $L, L^{\prime} \in \mathcal{G}_{2},\left[L \mid L^{\prime}\right]=0$ if and only if $L \cap L^{\prime} \neq \emptyset$.

The first point comes from the definition of the inner-product. The second point is the expansion of a $4 \times 4$ determinant by the Laplace's rule in terms of $2 \times 2$ minors. For the third point, remark that $L$ is of the form $a \wedge b, L^{\prime}$ of the form $c \wedge d$ and $L$ meets $L^{\prime}$ iff the 4 points $a, b, c, d$ are in a same plane : $\operatorname{det}(a, b, c, d)=0$. 


\section{Relations}

Let $\mathbf{k}\left[\mathcal{G}_{k}\right]$ be the space of algebraic functions on $\mathcal{G}_{k}$. They are polynomials of the coordinates $\mathbf{X}_{k}=\left\{\left[j_{1}, \ldots, j_{k}\right]\right.$, with $\left.0 \leq j_{1}<\cdots<j_{k} \leq n\right\}$. The identities satisfied by these variables form an ideal $I_{k}$ which is generated by the Plücker relations.

Moreover, there is an algorithmic way to reduce to a normal form the polynomials in these variables. It involves the structure of algebra with straightening laws of $\mathrm{k}\left[\mathcal{G}_{k}\right]$.

Definition B.2 - Let $\ll$ be the partial order on $\mathbf{X}_{k}$ such that

$$
\left[j_{1}, \ldots, j_{k}\right] \ll\left[j_{1}^{\prime}, \ldots, j_{k}^{\prime}\right] \text { iff } j_{1} \leq j_{1}^{\prime}, \ldots, j_{k} \leq j_{k}^{\prime} .
$$

We say that $m$ is a standard monomial for $\ll$ if it is of the form $m=x_{1} \cdots x_{k}$ with $x_{i} \in \mathbf{X}_{k}$ and $x_{1} \ll \cdots \ll x_{k}$. Let $S$ be a subset of the standard monomials for $\ll$.

Proposition B.3 - The set of standard monomials $S$ form a $\mathbf{k}$-basis of the algebra $\mathbf{k}\left[\mathcal{G}_{k}\right]$. Any polynomial in the variables $\mathcal{G}_{k}$ can be reduce to a normal form with the rules

$$
\begin{aligned}
& {\left[i_{1}, \ldots, i_{k}\right]\left[j_{1}, \ldots, j_{k}\right]} \\
& \quad \rightarrow-\epsilon(\sigma) \sum_{\sigma \in \mathcal{S} \backslash\{\text { id }\}}\left[i_{1}, \ldots, i_{l}, i_{l+1}^{\sigma}, \ldots, i_{k}^{\sigma}\right]\left[j_{1}^{\sigma}, \ldots, j_{l+1}^{\sigma}, j_{l+2}, \ldots, j_{k}\right]
\end{aligned}
$$

with $i_{1} \leq j_{1}, \ldots i_{l} \leq j_{l}$ but $i_{l+1}>j_{l+1}$ and where $\mathcal{S}$ is the set of permutations of $\left\{i_{l+1}, \ldots, i_{k}, j_{1}, \ldots, j_{l+1}\right\}$ such that $i_{l+1}^{\sigma}<\cdots<i_{k}^{\sigma}$ et $j_{1}^{\sigma}<\cdots<j_{l+1}^{\sigma}$.

This can be extended to determinants of different sizes. References for this proposition can be found in [4], [20].

\section{Intersection and Sum}

In order to represent intersection and sum of vector space, we introduce the following operator. For any elements $A=a_{1} \wedge \cdots \wedge a_{p} \in \wedge^{p} \mathbb{E}, B=b_{1} \wedge \cdots \wedge b_{q} \in$ $\wedge^{q} \mathbb{E}$

$$
A \Delta B=\sum_{\sigma} \epsilon(\sigma) a_{n-q+1}^{\sigma} \wedge \cdots \wedge a_{p}^{\sigma}\left|a_{1}^{\sigma}, \ldots, a_{n-q}^{\sigma}, b_{1}, \ldots, b_{q}\right|
$$

$\operatorname{RR} \mathrm{n}^{\circ} 2665$ 
if $p+q \geq n$ and 0 elsewhere.

This element of $\wedge^{p+q-n} \mathbb{E}$ represents the intersection of $\vec{A}$ and $\vec{B} \subset \mathbb{E}$ if it is a generic intersection (if the dimension of the intersection is $p+q-n$ ).

This is the usual Cayley operator defined in [2], [6]. It assumes implicitly that the intersection of the linear spaces is generic. For instance, in the space $\mathbb{P}_{3}$ the intersection of a line $a \wedge b$ with a plane $c \wedge d \wedge e$ is given by $a|b, c, d, e|-$ $b|a, c, d, e|$. With this representation, it is easy to see that it is an element of the line $\langle a, b\rangle$. Using Cramer's relation, we can also rewrite it as $-c|a, b, d, e|+$ $d|a, b, c, e|-e|a, b, c, d|$ which is typically an element of $\langle d, c, e\rangle$.

But in geometric problems, it is frequent that the intersection is not of the generic dimension. Think for instance to the intersection of two coplanar lines in a space of dimension $\geq 3$. So one needs to extend this operator defining $\Delta_{k}$ when the intersection is of dimension $p-k$. This can be done by changing the scalars and introducing "determinants" of different size $\left|a_{1}, \ldots, a_{k}\right|$ (for instance $\left|a_{1}, \ldots, a_{k}, t_{k+1}, \ldots, t_{n}\right|$ where $t_{i}$ are generic vectors of $\left.\mathbb{E}\right)$. See [21].

$$
A \triangle_{k} B=\sum_{\sigma} \epsilon(\sigma) a_{p-k+1}^{\sigma}, \ldots, a_{p}^{\sigma}\left|a_{1}^{\sigma} \wedge \cdots \wedge a_{p-k}^{\sigma} \wedge b_{1} \wedge \cdots \wedge b_{q}\right|
$$

In this case, it is also possible to define the operator "sum" as follows:

$$
A \nabla_{k} B=\sum_{\sigma} \epsilon(\sigma) a_{1}^{\sigma} \wedge \cdots \wedge a_{p-k}^{\sigma} \wedge b_{1} \wedge \cdots \wedge b_{q}\left|a_{p-k+1}^{\sigma}, \ldots, a_{p}^{\sigma}\right|
$$

This allows us to use Cramer's rule as follows. Suppose that we have a relation of the form $a \wedge a_{1} \wedge \cdots \wedge a_{d}=0$ where $a_{1} \wedge \ldots \wedge a_{d} \neq 0$. We would like to derive a relation on $a$. As $\left(a_{1}, \ldots, a_{d}\right) \mapsto\left|a_{1}, \ldots, a_{d}\right|$ is a multilinear alternate form, we can deduce that

$a\left|a_{1}, \ldots, a_{d}\right|-a_{1}\left|a_{2}, \ldots, a_{d}, a\right|+a_{2}\left|a_{1}, a_{3}, \ldots, a_{d}, a\right|+\cdots+(-1)^{d} a_{d}\left|a_{1}, \ldots, a_{d-1}, a\right|=0$.

If we are working in a projective space, as $\left|a_{1}, \ldots, a_{d}\right| \neq 0$, we can use the rule of substitution

$$
a \rightarrow a_{1}\left|a_{2}, \ldots, a_{d}, a\right|-a_{2}\left|a_{1}, a_{3}, \ldots, a_{d}, a\right|+\cdots+(-1)^{d-1} a_{d}\left|a_{1}, \ldots, a_{d-1}, a\right| .
$$




\section{Working in the dual}

One is often lead to work in the dual space $\hat{\mathbb{E}}$ of linear forms on $\mathbb{E}$. We can proceed in the same way as with $\mathbb{E}$, defining $\wedge \hat{\mathbb{E}}$, the Grassmannian of $\wedge \hat{\mathbb{E}}, \ldots$

The kernel of an element $H$ of $\hat{\mathbb{E}}$ is an hyperplane of $\mathbb{E}$. We also associate to the element $L=H_{1} \wedge \cdots \wedge H_{k}$, the linear space $\vec{L}$ of hyperplanes $H$ such that $H \wedge H_{1} \wedge \cdots \wedge H_{k}=0$. We denote by $\bar{L}$ the set of vectors which are in $\operatorname{ker}(H)$ for all $H \in \vec{L}$. It corresponds to the intersection of the vector spaces $\overline{H_{1}}, \ldots, \overline{H_{k}}$.

So the interpretation of the geometric operations have to be inverted. The sum (resp. intersection) of elements of $\hat{\mathbb{E}}$ corresponds to intersection (using the well-know relation $\left.(A+B)^{\perp}=A^{\perp} \cap B^{\perp}\right)$ (resp. sum) in $\mathbb{E}$.

If $H_{1}, H_{2}, H_{3}, H_{4}, H_{5}$ are planes of $\mathbb{P}_{3}$, then $H_{1} \wedge H_{2}\left(\right.$ resp. $\left.H_{3} \wedge H_{4} \wedge H_{5}\right)$ represents the intersection line (resp. point) of the two (resp. 3) planes and

$$
H_{1}\left|H_{2}, H_{3}, H_{4}, H_{5}\right|-H_{2}\left|H_{1}, H_{3}, H_{4}, H_{5}\right|
$$

is the plane passing through this line and this point.

\section{References}

[1] D.C. Ballard and C.M. Brown. Computer Vision. Prentice-Hall, Englewood Cliffs, New Jersey, 1982.

[2] M. Barnabei, A. Brini, and G.C. Rota. On the exterior calculus of invariant theory. J. of Algebra, 96:p 120-160, 1985.

[3] Stefan Carlsson. Multiple image invariance using the double algebra. In Joseph L. Mundy, Andrew Zissermann, and David Forsyth, editors, Applications of Invariance in Computer Vision, volume 825 of Lecture Notes in Computer Science. Springer-Verlag, 1994.

[4] C. DeConcini, D. Eisenbud, and C. Procesi. Hodge algebras. Astérisque, 91, 1982.

[5] J. A. Dieudonné. Eléments d'analyse, volume 3. GAUTHIER VILLARS, 1968.

$\mathrm{RR} \mathrm{n}^{\circ} 2665$ 
[6] P. Doubilet, G. C. Rota, and J. Stein. Foundations of Combinatorics IX: Combinatorial methods in Invariant Theory. Studies in Appl. Math., 53:185-216, 1974.

[7] Olivier Faugeras. Three-Dimensional Computer Vision: a Geometric Viewpoint. MIT Press, 1993.

[8] Olivier Faugeras and Luc Robert. What can two images tell us about a third one? The International Journal of Computer Vision, 1994. To appear. Also INRIA Technical report 2018.

[9] Olivier D. Faugeras. What can be seen in three dimensions with an uncalibrated stereo rig. In Giulio Sandini, editor, Proceedings of the 2nd European Conference on Computer Vision, pages 563-578. Springer-Verlag, Lecture Notes in Computer Science 588, May 1992.

[10] Olivier D. Faugeras, Tuan Luong, and Steven Maybank. Camera selfcalibration: theory and experiments. In Giulio Sandini, editor, Proceedings of the 2nd European Conference on Computer Vision, pages 321334. Springer-Verlag, Lecture Notes in Computer Science 588, 1992.

[11] Robert M. Haralick and Linda G. Shapiro. Computer and Robot Vision. Addison-Wesley, 1993.

[12] Richard Hartley. Lines and points in three views-an integrated approach. In Proc. Image Understanding Workshop, Monterey, California, November 1994.

[13] Richard Hartley, Rajiv Gupta, and Tom Chang. Stereo from Uncalibrated Cameras. In Proceedings of CVPR92, Champaign, Illinois, pages 761-764, June 1992.

[14] W. Hodge and D. Pedoe. Methods of algebraic geometry. Cambridge University Press, 1952.

[15] Berthold Klaus Paul Horn. Robot Vision. MIT Press, 1986. 
[16] Q.-T. Luong and T. Viéville. Canonic representations for the geometries of multiple projective views. In Jan-Olof Eklundh, editor, Proceedings of the 3rd European Conference on Computer Vision, pages 589-599, Vol. I. Springer-Verlag, Lecture Notes in Computer Science 800-801, 1994.

[17] Quang-Tuan Luong. Matrice Fondamentale et Calibration Visuelle sur l'Environnement-Vers une plus grande autonomie des systèmes robotiques. PhD thesis, Université de Paris-Sud, Centre d'Orsay, December 1992.

[18] Quang-Tuan Luong, Rachid Deriche, Olivier Faugeras, and Théodore Papadopoulo. On Determining the Fundamental Matrix: Analysis of Different Methods and Experimental Results. Technical Report 1894, INRIA, 1993.

[19] Quang-Tuan Luong and Olivier Faugeras. The Fundamental matrix: theory, algorithms, and stability analysis. The International Journal of Computer Vision, 1994. To appear.

[20] B. Mourrain. Approche effective de la théorie des invariants des groupes classiques. PhD thesis, Ecole Polytechnique, September 1991.

[21] B. Mourrain. Géométrie et interprétation générique ; un algorithme. In Effective Methods in Algebraic Geometry (MEGA), volume 94 of Progress in Math., pages 363-377. Birkhäuser, 1991.

[22] B. Mourrain. New aspects of geometrical calculus with invariants. Advances in Mathematics, 1994. to appear.

[23] B. Mourrain and N. Stolfi. Invariants methods in Discrete and Computational Geometry, chapter Computational Symbolic Geometry. Kluwer acad. pub., 1994. (to appear).

[24] J.G. Semple and G.T. Kneebone. Algebraic Projective Geometry. Oxford: Clarendon Press, 1952. Reprinted 1979.

[25] Amnon Shashua. Algebraic functions for recognition. IEEE Transactions on Pattern Analysis and Machine Intelligence, 1994. In press.

$\operatorname{RR} \mathrm{n}^{\circ} 2665$ 
[26] Amnon Shashua. Projective structure from uncalibrated images: structure from motion and recognition. IEEE Transactions on Pattern Analysis and Machine Intelligence, 16(8):778-790, 1994.

[27] Amnon Shashua and Michael Werman. Fundamental tensor: On the geometry of three perspective views. Technical report, The Hebrew University of Jerusalem, September 1994.

[28] Bernd Sturmfels. Algorithms in Invariant Theory. Springer-Verlag Wien New york, 1993.

[29] Bill Triggs. The Geometry of Projective Reconstruction I: Matching Constraints and the Joint Image. Technical report, LIFIA, INRIA Rhône Alpes, November 1994.

[30] Z. Zhang, R. Deriche, O. Faugeras, and Q.-T. Luong. A robust technique for matching two uncalibrated images through the recovery of the unknown epipolar geometry. Research Report 2273, INRIA Sophia-Antipolis, France, May 1994. submitted to Artificial Intelligence Journal.

[31] Andrew Zisserman and Steven Maybank. A Case Against Epipolar Geometry. In Joseph L. Mundy, Andrew Zissermann, and David Forsyth, editors, Applications of Invariance in Computer Vision, volume 825 of Lecture Notes in Computer Science. Springer-Verlag, 1994. 


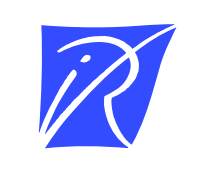

Unité de recherche INRIA Lorraine, Technopôle de Nancy-Brabois, Campus scientifique, 615 rue du Jardin Botanique, BP 101, 54600 VILLERS LÈS NANCY

Unité de recherche INRIA Rennes, Irisa, Campus universitaire de Beaulieu, 35042 RENNES Cedex

Unité de recherche INRIA Rhône-Alpes, 46 avenue Félix Viallet, 38031 GRENOBLE Cedex 1

Unité de recherche INRIA Rocquencourt, Domaine de Voluceau, Rocquencourt, BP 105, 78153 LE CHESNAY Cedex

Unité de recherche INRIA Sophia-Antipolis, 2004 route des Lucioles, BP 93, 06902 SOPHIA-ANTIPOLIS Cedex

Éditeur

INRIA, Domaine de Voluceau, Rocquencourt, BP 105, 78153 LE CHESNAY Cedex (France)

ISSN 0249-6399 